\title{
Postural Force Fields of the Human Arm and Their Role in Generating Multijoint Movements
}

\author{
Reza Shadmehr, Ferdinando A. Mussa-Ivaldi, and Emilio Bizzi \\ Department of Brain and Cognitive Sciences, Massachusetts Institute of Technology, Cambridge, Massachusetts 02139
}

\begin{abstract}
When a perturbation displaces the human hand from equilibrium, arm muscles respond by producing restoring forces. When a set of displacements are given at various directions from the same equilibrium position, the resulting restoring forces form a "postural force field." It is not known whether these postural forces are related to those generated when a reaching movement is executed. However, if a movement is a consequence of a shift of the equilibrium position of the hand toward the target, then, from the postural force field, predictions can be made regarding the nature of the elastic forces acting on the hand during the movement. We have taken the first steps in testing this hypothesis by measuring the postural force field of a subject's arm over relatively large distances, and comparing these forces with the static forces generated at the hand while the subject attempted a reaching movement.
\end{abstract}

Using a robot manipulandum, the hand was displaced at various directions from an equilibrium position. The measured restoring forces were fitted to a nonlinear model to define a postural force field for that equilibrium position. This field was used to predict elastic forces generated when the subject attempted to move the manipulandum from a point on the circumference of a circle to a target at its centerthe center corresponded to the equilibrium position at which the postural field was measured. In some of the movement trials, the manipulandum was locked during approximately the first $120 \mathrm{msec}$ of the program for motion and the resulting static "evoked" forces measured. We found that (1) the evoked forces did not point to the target, but were a function of the configuration of the arm and rotated with the shoulder joint, and (2) the magnitude of the evoked forces varied systematically, even though the movements were of the same magnitude. These patterns were remarkably similar to those observed in the postural forces.

Our results provide experimental evidence linking maintenance of posture in a multijoint system to that of generating a movement. The evidence is consistent with the hypothesis that the CNS programs a reaching movement by shifting the equilibrium position of the hand toward the target.

\footnotetext{
Received Mar. 18, 1992; revised June 23, 1992; accepted July 21, 1992.

This research was supported in part by grants from the NIH (NS09343 and AR26710) and the Office of Naval Research (N00014/88/K/0372). R.S. is recipient of a postdoctoral fellowship from the McDonnell-Pew Center for Cognitive Neurosciences at MIT. We thank J. Won, E. Fasse, and M. Cantor for their technical assistance, and are also grateful for stimulating discussions with Drs. T. Flash, S. Giszter, and N. Hogan.

Correspondence should be addressed to Dr. Reza Shadmehr, Department of Brain and Cognitive Sciences, Room E25-534, Massachusetts Institute of Technology, Cambridge, MA 02139.

Copyright (C) 1993 Society for Neuroscience $0270-6474 / 93 / 130045-18 \$ 05.00 / 0$
}

[Key words: postural force field, equilibrium point, arm posture, multijoint movement, arm stiffness, evoked forces, field interpolation, motor control]

The idea that a limb's posture is controlled through selection of the agonist and antagonist muscles' length-tension curves was first proposed by Feldman (1966). The notion of an "equilibrium position controller" was born from the observation that in a single-joint system, the length-tension properties of opposing muscles interact to stabilize the limb at some joint configuration - the limb resists displacement from this equilibrium position by producing restoring forces opposing the perturbation.

Feldman (1966) linked the process of postural control and movement generation by suggesting that the CNS might be generating a movement of the limb through selection of a set of length-tension properties of agonist and antagonist muscles such that the equilibrium position of the limb gradually shifts to the joint configuration corresponding to the target of the movement. This sequence of equilibrium positions is known as an "equilibrium trajectory." The essential characteristic of this hypothesis is that at every stage of the motor program, the control signal corresponds to a stable equilibrium position somewhere along the path connecting the start to the target configuration of the limb.

For moderate-speed single-joint movements, there is now a substantial body of evidence supporting the hypothesis that a movement is programmed by gradually shifting the equilibrium position of the limb to the target configuration (Bizzi et al., 1976, 1978, 1982, 1984; Kelso and Holt, 1980). In the case of multijoint movements, however, the question of whether a reaching task is executed as a result of a program that specifies such a trajectory remains unresolved. In a model of the human arm described by Flash (1987), the trajectory of the hand observed during certain reaching movements has been shown to be consistent with the predictions of the hypothesis. In these simulations, a movement was produced as a consequence of a motor program that specified a trajectory of equilibrium points for the hand along a straight line from the start to the target position. However, it has been suggested (Hasan, 1991; Smith and Humphrey, 1991) that simulation paradigms may not provide an adequate measure of this hypothesis because of the large number of uncertain parameters related to the mechanical impedance of the arm in its multijoint configuration (Hogan, 1985). Nevertheless, it is worth noting that the results of the simulations performed by Flash (1987) were critically dependent on only one assumption, namely, that the motor program for reaching could be described by a voluntary shift in the equilibrium position of the hand such that at any point in time, the static 


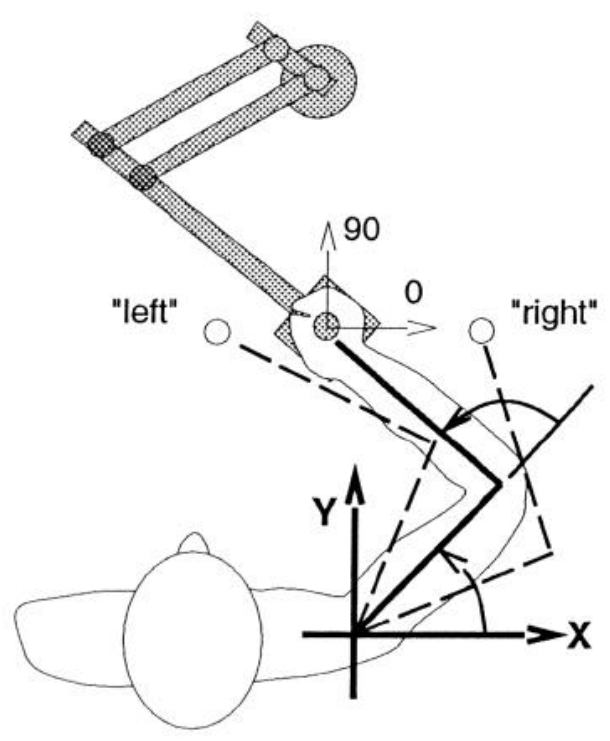

Figure 1. Sketch of the manipulandum and the experimental setup. The manipulandum is a two-joint planar robot with a force transducer mounted on its end-effector. In the postural force field measurements, the manipulandum would displace the subject's hand, while in the movement trials, the subject would move the manipulandum. These measurements were performed at two positions in the work space (broken lines). For a typical subject, at the "left" position the shoulder and elbow joint angles were $57^{\circ}$ and $99^{\circ}$, respectively, while at the "right" position these angles were $18^{\circ}$ and $88^{\circ}$. Lengths of the forearm and the upper arm for this subject were 33.5 and $33.8 \mathrm{~cm}$, respectively. The coordinate system used to specify a direction is shown: a target at 3:00 was at $0^{\circ}, 12: 00$ at $90^{\circ}$, and so on.

component of the forces acting on the hand were the same as those obtained when the hand was displaced from that equilibrium position. This is a nontrivial assumption because the static response of the human arm (in its multijoint configuration) when the hand is passively displaced by a small distance from equilibrium is much more complicated than that observed from a single-joint system: in a stable single-joint system, regardless of the number of muscles or complexity of the their geometry, restoring torques are always opposite to the direction of the displacement; in a multijoint system, however, displacement of one joint may result in development of torque at not only the affected joint, but also neighboring joints as well. For example, this may be due to the effect of mechanical interaction between the joints through the action of double-joint muscles (cf. Hogan, 1985), or the action of heterogenic reflexes (Nichols, 1989).

The first attempt at understanding multijoint behavior of the human arm near equilibrium was performed by Mussa-Ivaldi et al. (1985). In these experiments, the hand was displaced by a few millimeters from equilibrium and the restoring forces measured. An essential finding of this work was that the direction of the restoring force was not, in general, opposite to the direction of the displacement. From this we can infer that based on the equilibrium trajectory hypothesis, for a multijoint reaching movement, if the equilibrium position of the hand were to be voluntarily shifted toward a target, then the static forces produced at the hand should not point to the target. In fact, the restoring forces measured about an equilibrium position can provide a strong prediction regarding the nature of the elastic forces that should be produced if a reaching movement is generated through a shift of the hand's equilibrium position toward a target.

In particular, consider the restoring forces that result when the resting hand is displaced systematically from the center of a circle to points that lie about its circumference. According to the equilibrium point hypothesis, in order to make a reaching movement from a point on the circumference of this circle to a target at its center, the static forces produced at the hand should be directly related to the restoring forces measured during maintenance of posture. Measurement and comparison of these two forces are the thrust of the present study.

Our intention is to ask whether there is a link between maintenance of posture and the act of generating a multijoint movement. To that end, in the present study we have designed a method to measure the restoring forces for relatively large displacements of the hand from equilibrium. These postural forces were then compared to forces measured during a task in which the subject was to move the hand to a target. During randomly selected movement trials, as soon as the target was presented, we prevented the hand from moving and measured the resulting static "evoked" forces. When the postural forces were compared to the evoked forces, we found remarkable similarities in the directions and relative magnitudes of the two data sets. These findings provide experimental evidence that links maintenance of posture to generation of movement, and is consistent with the predictions of the hypothesis that a reaching movement is programmed through a shift of the equilibrium position of the hand toward the target.

\section{Materials and Methods}

Seven right-handed subjects with no known history of neuromuscular disease, ranging in age from 24 to 38 years, participated in this study. A schematic of the measurement apparatus is shown in Figure 1. Subjects were seated on a chair that was bolted onto an adjustable positioning mechanism and were instructed to grip the handle of a planar manipulandum with their right hand. Their shoulder was restrained by a harness belt, their right elbow was supported in the horizontal plane by a rope attached to the ceiling, and their wrist on the right hand was wrapped in a brace to prevent rotation.

\section{Apparatus}

The manipulandum is a two-joint, lightweight planar robot with a sixaxis force-torque transducer mounted on its end-effector (the handle). The shoulder and elbow links of the robot have, respectively, lengths of 23 and $20 \mathrm{~cm}$ and moments of inertia of 0.0195 and $0.0037 \mathrm{Kg} \cdot \mathrm{m}^{2}$. At the configuration tested (center of the work space), the inertia of the robot as expressed in end-point coordinates (so-called mass matrix) was estimated to have a determinant of $0.15 \mathrm{Kg}^{2}$, which is approximately an order of magnitude smaller than that of a typical human arm.

Two optical encoders, mounted on the axes of the mechanical joints, provided position information, while two tachometers provided joint velocity signals. The torque motors (mounted at the base of the robot) were connected independently to each joint. Furthermore, each joint was equipped with a brake mechanism that could be activated to lock the manipulandum. The apparatus also included a video display monitor that in some instances was used to provide feedback to the subject regarding position of the handle relative to a specified target.

A microcomputer collected position, velocity, and force information at a rate of $100 \mathrm{~Hz}$, and controlled the torque motors and the brakes. In order to give the robot a desired stiffness and viscosity, and to allow for servoing of the subject's arm to a desired position, we used the following linear control law:

$$
\mathbf{f}=K_{m}\left(\mathbf{x}-\mathbf{x}_{d}\right)-B_{m} \dot{\mathbf{x}},
$$

where $f$ is the effective force vector at the end-effector produced by the torque motors, $\mathbf{x}$ and $\mathbf{x}$ are the current position and velocity vectors of the end-effector, $\mathbf{x}_{d}$ is its desired position, and $K_{m}$ and $B_{m}$ are the programmed stiffness and viscosity matrices for the manipulandum. The purpose of this control law was to provide a mechanism for displacing the arm of the subject along a desired path. In all experiments, we used the following stiffness and viscous parameters: 


$$
\begin{aligned}
& K_{m}=\left[\begin{array}{rr}
-0.7 & 0.0 \\
0.0 & -0.7
\end{array}\right] \mathrm{N} / \mathrm{mm}, \\
& B_{m}=\left[\begin{array}{rr}
-19 & 0.0 \\
0.0 & -19
\end{array}\right] \quad \mathrm{N} /(\mathrm{m} / \mathrm{sec}) .
\end{aligned}
$$

These parameters were chosen empirically so to maintain the manipulandum's stability while providing the largest apparent stiffness compatible with the operating range of the torque motors $( \pm 8.9 \mathrm{~N} \cdot \mathrm{m})$.

\section{Experimental procedures}

Each subject participated in two experiments. In experiment 1, restoring forces generated by the subject while the hand was displaced from equilibrium were measured. The displacement was from the center to various points along the circumference of a circle, where the center corresponded to the equilibrium position of the hand. The restoring forces for a set of displacements were used to define a "postural force field." In experiment 2 , subjects were instructed to move the manipulandum from a point at the circumference of the same circle to a target at its center. We measured the static forces produced at the hand in response to presentation of a target in the context of a reaching task.

Measuring the postural force field. The measurement of the postural force field can be regarded as an extension of the technique introduced by Mussa-Ivaldi et al. (1985) to measure the postural stiffness. After the subjects were positioned in front of the manipulandum, they were instructed to grip the handle, to place it at a specified location in the work space, and to close their eyes. They were told that the manipulandum would attempt to displace their arm slowly, and were asked "not to intervene voluntarily." The perturbation was programmed as a gradual change in the nominal position of the handle, that is, $\mathbf{x}_{d}$ in Equation 1. At the beginning of the perturbation, the nominal position of the handle was set to be equal to the actual position: $\mathbf{x}_{d}=\mathbf{x}$. From this initial position, $\mathbf{x}_{d}$ was moved $5.0 \mathrm{~cm}$ over a period of $8.5 \mathrm{sec}$ (at constant velocity) along a straight line in a specified direction. After the $8.5 \mathrm{sec}$ ramp period, $\mathbf{x}_{d}$ was kept constant for $0.4 \mathrm{sec}$ and then the torque motors were turned off, allowing the arm/manipulandum system to move freely. This measurement was repeated for 24 directions (spanning $360^{\circ}$ ), presented in a random order.

As the manipulandum displaced the hand, the subject's arm produced gradually increasing restoring forces on the manipulandum. Figure 2 is an example of one such perturbation and the forces measured at the handle: Figure $2 A$ shows the displacement to the hand and Figure $2 B$ is a plot of the resulting restoring forces. The solid lines represent displacement and forces along the $\mathrm{x}$-direction, while the broken lines represent displacement and forces along the $y$-direction. The coordinate system was centered at the subject's shoulder joint (Fig. 1). In this case, the manipulandum attempted to move the subject's arm along a line directed at $75^{\circ}$. Note that there is a smooth and continuous increase in both components of the force as the arm is displaced, and that the arm returns to a location very close to the initial posture after release.

Approximately $2 \mathrm{sec}$ after the perturbation had completed and the hand had returned to its initial position, a second perturbation along a different direction was given. Approximately $30 \mathrm{sec}$ after the completion of the 24 perturbations, a second and then a third set of perturbations (each consisting of 24 perturbations) would follow. The purpose of this repetition was to quantify the variability of the measured fields.

From these force-position data, we derived a postural force field corresponding to the particular configuration of the arm at which restoring forces were measured. A force field was measured in each subject at two different arm positions in the work space. This is indicated in the schematic of Figure 1. In order to prevent any artifacts arising from changes in the configuration of the apparatus, we kept the handle at the same position relative to the base of the manipulandum and moved the subject on the adjustable chair. We will refer to these locations as the "right" and "left" arm positions.

Evoked forces. The objective of this second part of the experiment was to quantify the static forces produced by the subject when the task was to move the manipulandum's handle to a target. The subjects were instructed to grasp the handle of the manipulandum and to move it such that the cursor displayed on the video monitor (representing the current position of the handle) was placed within a target square. The square represented an area of $4 \times 4 \mathrm{~mm}$ in the work space. Once the starting location was reached, a target was presented. The subject was asked to move to this target. The instructions were to "make a single and uncorrected movement to reach the target square."
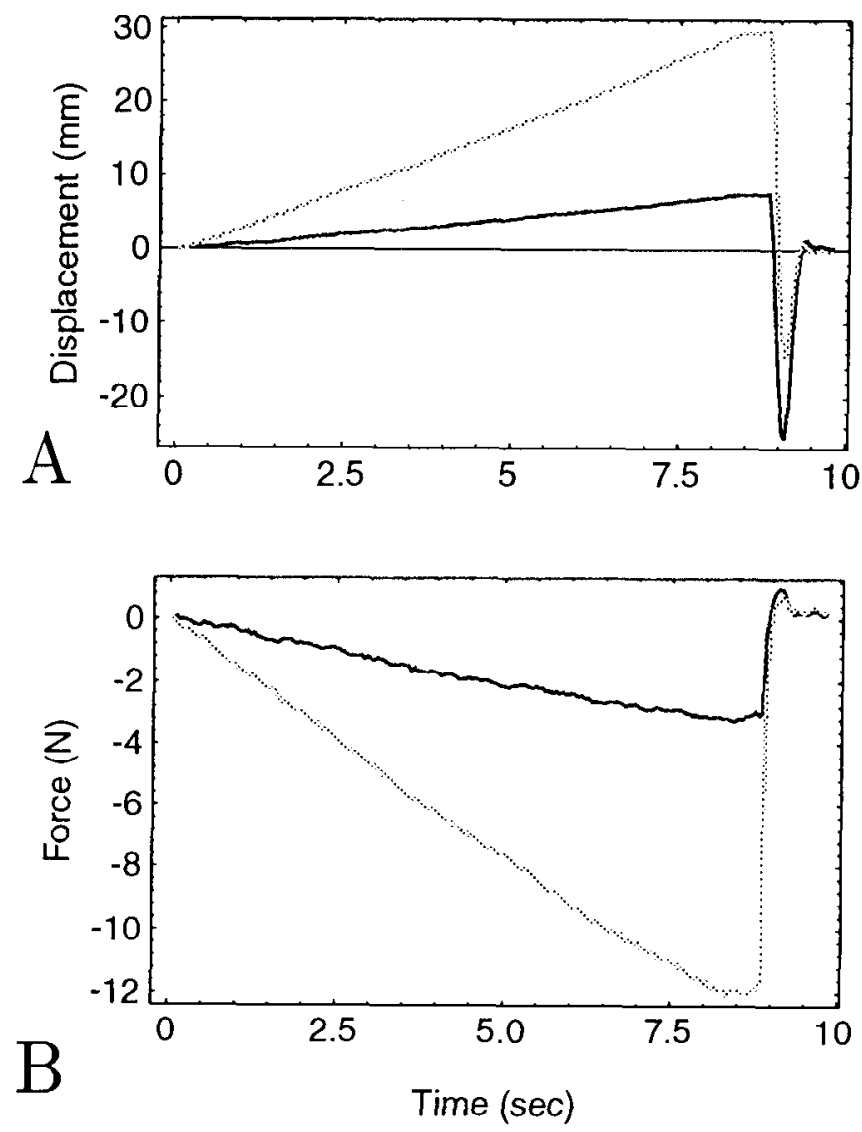

Figure 2. Restoring forces for a two-dimensional displacement of the human arm. The perturbation was along $75^{\circ}$, and attempted to displace the hand by $50 \mathrm{~mm}$ during $8.5 \mathrm{sec}$ (constant velocity). $A$, Hand position with respect to initial position. Solid and broken lines are the position components along the $\mathrm{x}$ - and $\mathrm{y}$-axcs, respectivcly. $B$, Restoring forces measured at the hand. Solid and broken lines are $f_{x}$ and $f_{y}$, respectively.

We specified the starting position for each movement on the circumference of a circle of $40 \mathrm{~mm}$ radius. The target position for all movements was the center of this circle, and corresponded to the postural location used in the first experiment. The initial positions were distributed at $45^{\circ}$ intervals. Therefore, the movement trials converged on a single final position from eight different initial positions.

In order to reach the target, the subject exerted a force on the handle of the manipulandum, which also housed the force transducer assembly. During a movement, the forces measured by the transducer reflected the dynamic interactions between the subject and the manipulandum. These forces were influenced by the inertial, viscous, and elastic properties of the subject's arm and by the dynamics of the manipulandum. However, by locking the arm at the onset of the movement, it was possible to remove the inertial and viscous components. Thus, we implemented the following procedure aimed at measuring the elastic forces generated by a subject at the onset of movement. In randomly selected trials, as soon as the target was displayed on the monitor, the brakes were activated on the manipulandum, effectively locking its end-position. The brakes exerted their action for $300 \mathrm{msec}$ only. The forces measured during this period are static in nature, and will be referred to as "evoked forces" to distinguish them from the static forces measured in the postural trials.

As noted, movements were made from eight different initial positions toward the same target. The subject repeated movements from each of the initial positions 20 times. In eight randomly selected trials from each initial position, the brakes were applied. The subject made these movements at the two different arm positions corresponding to the left and right configurations in Figure 1. Thus, we obtained evoked force data for a total of 128 movement trials.

We noted that engaging the brakes produced a slight clicking noise that could have possibly aided the subject in anticipating a braking of the manipulandum as the target was shown. Since disengagement of the 

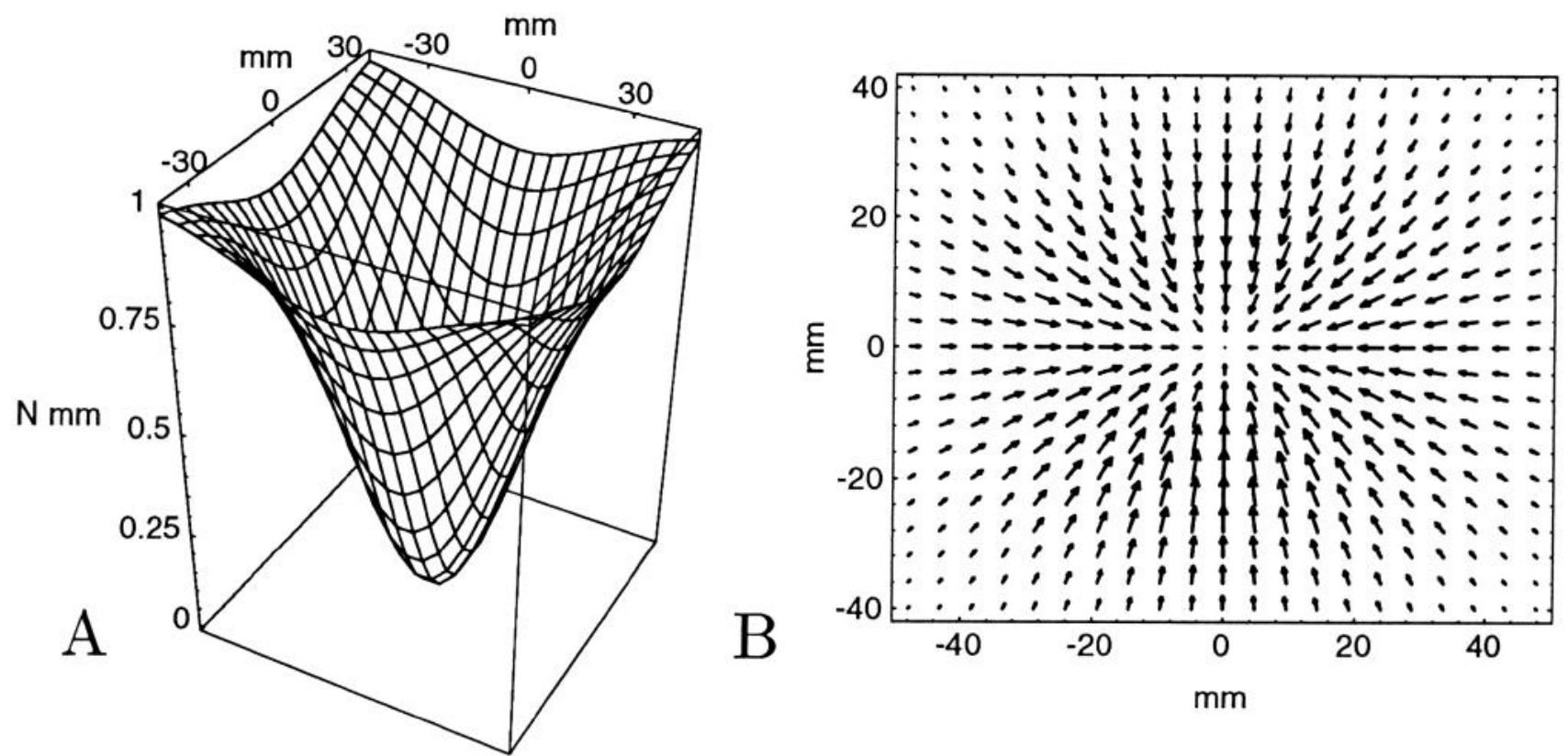

Figure 3. Example of the basis function and the corresponding basis field used in approximating the measured postural forces. $A$, The basis function $G\left(\mathbf{x}, \mathbf{x}_{i}\right)$, plotted for $\mathbf{x}_{i}=(0,0), \sigma_{x}=24$, and $\sigma_{y}=20 \mathrm{~mm}$ (see Eq. 5). B, The basis field, that is, the gradient of the function plotted in $A$.

brakes was inaudible, in trials for which the brakes were not to be applied for $300 \mathrm{msec}$, the brakes were turned on and then off as quickly as possible (a latency of approximately $15 \mathrm{msec}$ ). This ensured that all movement trials began with display of the target and a clicking noise of the brakes, even though only in some of the trials the brakes were kept on to measure the evoked forces.

\section{Data analysis and modeling}

In the case of the movement trials, we were interested in the forces produced by the subject as a function of target direction. Since significant forces did not develop until 170-200 msec after presentation of the target, we represented the evoked forces produced by the subject for that trial by computing an average force vector during the period between 200 and $300 \mathrm{msec}$ after the target was presented. However, in the rare event (less than $2 \%$ of the trials) that forces did not develop until less than $100 \mathrm{msec}$ before the release of the brakes, the trial was not used. For the eight times that the same target was presented, the mean force direction and magnitude along with the corresponding standard deviations were calculated to represent the force produced for a given target direction, at a given configuration of the arm.

For the analysis of the postural forces, a method had to be developed to address the following problem: Since the measured forces provided information for a finite set of points in the vicinity of the equilibrium posture (in approximately a $3-5 \mathrm{~cm}$ radius), we needed a way to use this data to predict what the forces would be at positions that were within this radius but for which no data point was available. Furthermore, since the postural forces were measured over three separate trials, it was necessary to have a means for comparing them. We addressed these issues by fitting the restoring forces to a nonlinear model that was our representation of the postural field. The procedure for approximating these fields is explained next.

Force field approximation. Here we describe a technique for approximating a force field (a more detailed account of this technique can be found in Mussa-Ivaldi et al., 1991). Our goal is to fit a function to the measured postural forces such that the data generated from the measurements and the forces produced by the approximated field are statistically consistent with a single distribution function (von Mises, 1964).

We solved this approximation problem by fitting the measured forces to a collection of predefined fields - each of these predefined fields was called a "basis field." Inherent in our procedure are two assumptions: (1) that the measured forces can be reproduced by summing a sufficient number of basis fields, and (2) that the measured forces belong to an integrable field that is the gradient of some scalar potential. The first assumption was found to be justified empirically through statistical comparison of the measured and approximated data. The second assumption is based on the fact that for perturbations of relatively smal magnitude, Mussa-Ivaldi et al. (1985) have shown that the restoring forces are conservative, meaning that the arm as a whole behaves as a spring-like system (Hogan, 1985), implying that a scalar potential function can be defined.

The statement of the problem is as follows. The data in a set of perturbations are samples of an unknown vector field $\mathbf{F}$. This field is a map defined by the following:

$$
\begin{aligned}
\mathbf{F}: \quad \mathbf{x} & \rightarrow \mathbf{f} ; \\
& \left\{\mathbf{f}=\left(f_{x}, f_{y}\right) \mid f_{x}=F_{x}(\mathbf{x}), f_{y}=F_{y}(\mathbf{x}) ; f_{x}, f_{y} \in R ; \mathbf{x}=(x, y)\right\} .
\end{aligned}
$$

We have some $d$ samples of $\mathbf{F},\left\{\mathbf{f}^{1}, \mathbf{f}^{2}, \ldots, \mathbf{f}^{d}\right\}$, obtained at a set of distinct locations $\left\{\tilde{\mathbf{x}}^{1}, \tilde{\mathbf{x}}^{2}, \ldots, \tilde{\mathbf{X}}^{d}\right\}$. The question is how to construct an estimate of $\mathbf{F}$ from the collected data points. Clearly, one approach is to operate on the single vector components $f_{x}$ and $f_{y}$ separately, treating them as unstructured collections of scalar entities. Another approach is to use basic computational elements that themselves are endowed with vector properties. The advantage of this latter approach is that a coordinate invariant description of the results can be achieved: consider that the field $\mathbf{F}$ is integrable (or conservative) if and only if a scalar potential function $U(\mathbf{x})$ can be defined over $R^{2}$ such that

$$
\mathbf{f}(\mathbf{x})=\left.\left(\frac{\partial U}{\partial x_{1}}, \frac{\partial U}{\partial x_{2}}\right)\right|_{\mathbf{x}} .
$$

Let us assume that the potential function $U(\mathbf{x})$ is approximated by a set of $k$ basis functions (Powell 1987, Poggio and Girosi, 1990), $G_{i}=G(\mathbf{x}$, $\left.\mathbf{x}_{i}\right)$ with $(i=1, \ldots, k)$ as

$$
U(\mathbf{x}) \sim \sum_{i=1}^{k} c_{i} G\left(\mathbf{x}, \mathbf{x}_{i}\right),
$$

where each point $\mathbf{x}_{i}$ is the "center" location for its basis function $G_{i}$. Accordingly, the vector field, $\mathbf{F}(\mathbf{x})$, can be approximated by a set of $k$ basis fields, $\varphi_{i}$. These basis fields are obtained from the corresponding basis functions as

$$
\boldsymbol{\varphi}_{i}(\mathbf{x})=\left(\varphi_{x}^{i}(\mathbf{x}), \varphi_{y}^{i}(\mathbf{x})\right)=\left(\frac{\partial G_{i}(x, y)}{\partial x}, \frac{\partial G_{i}(x, y)}{\partial y}\right) .
$$

The vector field $\mathbf{F}$ is approximated by summing a set of $k$ basis fields of Eq. (3) with the same coefficients $c_{i}$ that were introduced in Eq. (2): 


$$
\mathbf{F}-\sum_{i=1}^{k} c_{i} \varphi^{i}
$$

We used bivariate Gaussians as basis functions:

$$
G_{i}=\exp \left(-\frac{\left(x-x_{i}\right)^{2}}{2 \sigma_{i}^{2}}-\frac{\left(y-y_{i}\right)^{2}}{2 \sigma_{i}^{2}}\right)
$$

An example of the basis function $G_{i}$ is shown in Figure $3 A$. In Figure $3 B$ we have the corresponding basis field $\varphi$. For our purpose, the problem of field approximation is to find a coefficient $c_{i}$ for each basis field $\varphi$ so that when all basis fields are added, the sum is a field that is statistically consistent with the measured force field. The procedure that we followed for solving for each of the basis field coefficients is outlined in the next section.

Basis field coefficients. One way to solve for the coefficients $c_{i}$ for each of the $k$ basis fields in Equation 4 is to minimize the squared error between the approximated and measured fields. Solving for the coefficients $c_{i}$ is simple because Equation 4 is a linear transformation. Let us represent our measured data by a vector $\mathbf{p}$, such that $\mathbf{p}=\left(\mathbf{f}_{x}^{1}, \ldots, f_{x}^{d}\right.$, $\left.f_{v}^{\prime}, \ldots, f_{v}^{d}\right)$. This vector has $2 \times d$ components and is related to the array of coefficients $\mathbf{c}=\left(c_{1}, \ldots, c_{k}\right)$ by the linear transformation

$$
\mathbf{p}=\boldsymbol{\Phi} \mathbf{c}
$$

The matrix $\Phi$ is

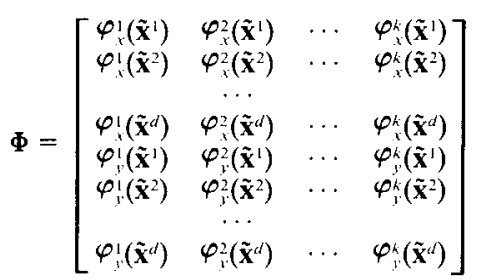

In the linear transformation of Equation 6 , we can uniquely solve for c when the number of basis fields, $k$, is $2 \times d$ and $\boldsymbol{\Phi}$ is not singular. If $k<2 \times d$, then the choice of the pseudoinverse $\boldsymbol{\Phi}^{+}=\left(\boldsymbol{\Phi}^{7} \boldsymbol{\Phi}\right)^{-1} \boldsymbol{\Phi}^{7}$ minimizes the square of errors, $\|\boldsymbol{\Phi} \mathbf{c}-\mathbf{p}\|^{2}$, so that we have $\mathbf{c}=\boldsymbol{\Phi}^{+} \mathbf{p}$. In this study, the number of basis fields used was significantly less than the number of data points; that is, $k \ll d$. Therefore, the pseudoinverse technique was used to solve for $\mathbf{c}$.

Statistical analysis of the model. Initially, we quantified how well the measured postural forces were represented by our field approximation technique. Once this approximation was shown to be statistically consistent with the measured data, we used the model to predict the evoked forces, assuming that the equilibrium position had moved toward the target. Finally, we tested the hypothesis that postural forces and evoked forces are consistent with the same distribution. Each phase of this procedure is described herein.

In approximating the postural forces through addition of a set of basis fields, we needed to decide (1) how many basis fields to use and (2) where to put the center of each basis field. As in any approximation task, the more degrees of freedom one has in the model, that is, the more basis fields one has, the more precisely one can approximate the data. However, the drawback of adding degrees of freedom is a loss of "smoothness" in the resulting field, particularly if the measured data are noisy. Therefore, it is desirable to use the least number of basis fields that satisfies an objective measure of error.

One way to determine how many basis fields are sufficient for the approximation problem is through a statistical test of how well the measured forces and the approximated field match. The Kolmogorov-Smirnov criterion is a nonparametric method to perform this comparison (von Mises, 1964; Hoel et al., 1971). The procedure is first to construct an unbiased estimator of the measured data's cumulative probability density (c.p.d.), and then to look at the c.p.d. for the approximated data and calculate the maximum value of the absolute difference between the two. This number can then be used to give a significance measure for certainty of the null hypothesis, that the measured and the approximated data sets are drawn from the same c.p.d.

The procedure that we used to decide on the location of the basis fields was as follows. Since the work space dimensions spanned by our force data were within a rectangle of $120 \times 100 \mathrm{~mm}$, we positioned the center $\mathbf{x}_{i}=\left(x_{i}, y_{i}\right)$ of the basis field $\varphi^{\text {i }}$ on a rectangular grid based on the following rule. Assuming that the number of basis fields that we chose to use is $k=k_{r} \times k_{c}$, where $k_{r}$ and $k_{c}$ are the number of columns and rows in the rectangular grid of the basis fields, then $x_{i}=-60+$ $i 60 / k_{i}$, and $y_{i}=-50+i 50 / k_{r}$ (both in millimeters).

The rule used to decide on the standard deviation of the bivariate Gaussian of Equation 5 depended on the number of basis fields as well. Our criterion was to ensure that all centers were located within one standard deviation from each other, ensuring a high level of interaction between neighboring basis fields. This interaction is important because the contribution of a basis field is always zero at the location of its center. Therefore, the $\sigma$ variables in Equation 5 were set as follows: $\sigma_{x}$ $=120 / k_{i}$, and $\sigma_{i}=100 / k_{r}$.

The approximated field was used to predict the evoked forces during the movement task, that is, to test the hypothesis that the evoked forces were due to a displacement of the equilibrium position of the hand toward the target. The predicted and measured evoked forces were compared by (1) computing an average error in matching of the data sets, and (2) computing a statistical measure of whether the two are consistent with the same distribution (the Kolmogorov-Smimov criterion).

\section{Results}

The equilibrium point hypothesis predicts a simple relationship between the elastic forces generated during the execution of a movement and the forces measured during maintenance of posture. According to this hypothesis, the CNS executes a movement by gradually shifting the equilibrium posture from the starting position to the final target (Bizzi et al., 1984). Thus, once one knows the field of static forces that are generated when the hand is displaced from equilibrium, one should also be able to predict the static forces generated as the subject attempts to move to a target at that equilibrium position.

In this study, we displaced the arm by a relatively large distance from equilibrium $(35-45 \mathrm{~mm})$, which resulted in significant restoring forces $(9-15 \mathrm{~N}$ ) (the displacements and restoring forces are approximately an order of magnitude larger than thosc applied in Mussa-Ivaldi et al., 1985). Our purpose was to ascertain whether there was a relationship between the postural forces developed over a distance of $40 \mathrm{~mm}$, and the static forces produced for a movement to a target at the same distance. The target of the movement was always situated at the center of the postural force field (the equilibrium position), and the starting position of the movements were always from points on the circumference of a circle of $40 \mathrm{~mm}$ in radius. This allowed for a direct comparison between the postural forces when the hand was displaced from the center to the circumference of the circle, and the evoked forces when a movement was generated from a point on the circumference to a target at the center of the circle.

\section{Postural forces}

Typical postural forces measured at the two arm positions are shown in Figure 4. These figures were constructed by plotting the measured restoring force vector as the hand was slowly displaced from the center to the periphery. Each displacement lasted $8.5 \mathrm{sec}$, and the forces in these plots represent the raw restoring forces developed at $0.50,1.50, \ldots, 8.50 \mathrm{sec}$ into the displacement.

Clearly, the manipulandum displaced the hand farther in some directions than others. I his effect was by design: the manipulandum was equally stiff in all directions (note that the matrix $K_{m}$, i.c., the manipulandum's stiffness, describes an isotropic field in Eq. 1), while the human arm was more stiff in some directions than in others. This meant that when the robot and the human arm interacted, the same force produced by the robot would tend to displace the human arm farther in directions for which the arm was least stiff, as compared to a perpendicular direction. From Figure 4, we note that (1) in general, forces were 

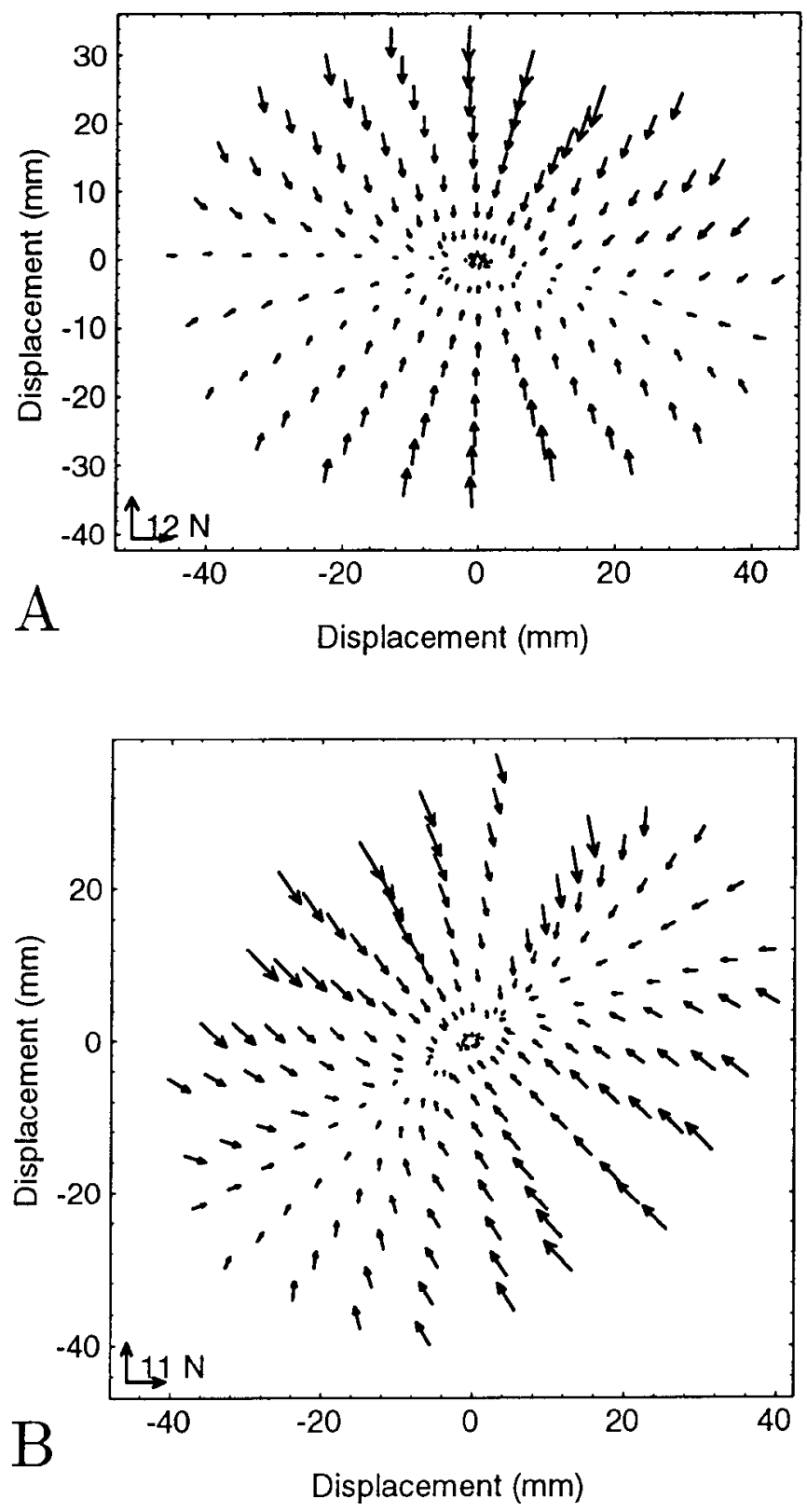

Figure 4. Measured postural force field for subject 1 . The arm is displaced from the center to the periphery. Restoring force vectors are shown at every $1 \mathrm{sec}$ interval. $A$, The arm is in the "right" position. $B$, The arm is in the "left" position.

not aligned along the direction of the displacement, (2) magnitude of the restoring forces was not equal for equal displacements from equilibrium, and (3) the field was shaped considerably differently at the two configurations of the arm.

We found that the general pattern of forces describing the postural fields at the two configurations of the arm were in agreement with the results of Mussa-Ivaldi et al. (1985), where it was found that for small displacements of the hand (about 4 $\mathrm{mm}$ ), the human arm was (1) most stiff along a line approximately connecting the hand to the shoulder, and (2) spring-like, that is, with a symmetric stiffness matrix. In order to compare the measured fields with the results of Mussa-Ivaldi et al. (1985), we fitted the postural forces of each subject to a series of stiffness matrices. The restoring forces for displacements of $0-1,1-2$,
2-3, and 3-4 cm were each fitted to a stiffness matrix: $\mathbf{f}=\mathbf{K}$ $d \mathbf{x}$, where $\mathbf{K}$ represented the stiffness of the limb. Typical results after fitting the stiffness matrices to the restoring forces are plotted in Figure 5 for the two configurations of the arm in the same subject. Each ellipse represents the stiffness at a given displacement from equilibrium. The principle axis of each ellipse corresponds to the direction of maximum stiffness of the arm, while the area of the ellipse is the determinant of the stiffness matrix. We found that (1) the direction of maximum stiffness was directed approximately toward the shoulder for both the "left" and the "right" configurations where a postural field was measured; (2) the stiffness matrix computed near equilibrium (for displacements of up to $1 \mathrm{~cm}$ ) was essentially symmetric: the antisymmetric component of the stiffness matrix was in all measurements contributing to less than $4.5 \%$ of the postural field (as estimated by the size of the determinant of the antisymmetric component of the stiffness matrix vs that of its symmetric component); and (3) there was a gradual reduction in the size of the stiffness matrices (represented by the area of the ellipses) as the robot displaced the subject's hand farther from its equilibrium position (the smallest ellipses in Fig. 5 correspond to the largest displacements). Therefore, the arm hecame less stiff as the hand was displaced farther away from equilibrium.

The first two observations are in agreement with the results of Mussa-Ivaldi et al. (1985). The third observation, however, is unique to the present study and illustrates that for our relatively large displacements from equilibrium, the use of a linear model (i.e., a single stiffness matrix) would be inadequate to describe the measured postural field. In fact, it is possible to illustrate other instances that suggest that the postural field consists of forces that are nonlinearly related to displacement from equilibrium. For example, in Figure $4 A$, note that when the hand is displaced at $0^{\circ}$, the restoring forces are not opposite to the forces that are produced when the hand is displaced at $180^{\circ}$.

In order to approximate the behavior of the arm near equilibrium, the postural forces were fitted to a nonlincar model that consisted of a set of predefined fields. It was necessary to approximate the force field so that we could make a quantitative assessment of the hypothesis that the forces evoked in response to a reaching target were in fact consistent with those measured during maintenance of posture. The model of the postural field allowed us to compare not only the direction of the postural forces to the evoked forces, but also their relative magnitudes. The results of this modeling effort are described below.

\section{Nonlinear modeling of the postural fields}

We have assumed that the measured restoring forces are samples of an underlying continuous force field that is produced by recruitment of muscles as the hand is displaced from an equilibrium position. The existence of such a continuous field is strongly suggested by the fact that although displacements were given at a random order in various directions, the resulting restoring forces were quite similar for similar displacements. This indicates that at some abstract level, the CNS is specifying an equilibrium position, as well as how the limb should behave if displaced from this equilibrium position. Because there is a postural field, rather than a collection of unrelated restoring forces, this representation in the CNS must remain constant over the period of mcasurement of the field. To approximate this field from the measured data points, for example, points in Figure 4, we chose a mathematical model consisting of a weighted sum of a set of predefined continuous fields. We call these "basis fields" since 


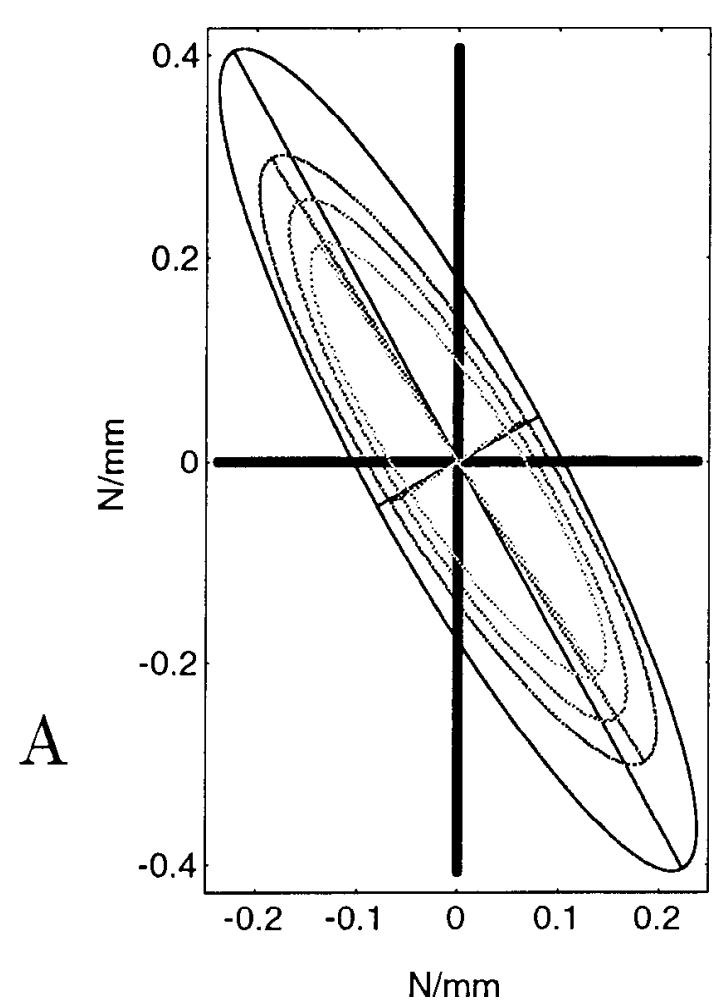

their role in the vector approximation problem is equivalent to the role of basis functions in a scalar approximation problem.

As an example, let us describe the field in Figure $4 A$ in terms of a sum of basis fields. The first question was how many basis fields to use to approximate the data. The procedure used to decide on the number of basis fields was as follows. We began with two basis fields and solved for their coefficients $c_{1}$ and $c_{2}$. We then increased the number of basis fields (see Materials and Methods) and computed the average absolute error between the measured field and the approximated field along the $x$ - and $y$-axes. Thus, we obtained an average error vector whose magnitude is plotted as a function of the number of basis fields in Figure 6. This figure also indicates the instances when the Kolmogorov_Smirnov test indicated that the approximation and the measured data were statistically consistent with each other. Using this method, we chose a conservative estimate of 25 basis fields to reproduce the measured force vectors in Figure $4 A$ accurately. Average absolute error for this approximation was $3.3 \%$ and $3.5 \%$ of the maximum $f_{x}$ and $f_{y}$ measured. This maximum force was measured at the boundary of the postural field, where we were interested in generating movements from.

The coefficients of these 25 centers, along with the location of each center, are given in Table 1. This table also gives the coefficients of the centers for the basis fields fitted to the data in Figure $4 B$. In both cases, based on the Kolmogorov-Smirnov criterion, the measured and approximated data sets were statistically consistent each other $(p=0.05)$. In general, we found that 25 basis fields provided a statistically sufficient approximation of all measured data sets in all subjects. Average absolute error for approximating the measured fields in all subjects was less than $5 \%$ of the maximum force, and in no case was the correlation coefficient $r^{2}$ less than 0.95 .

A direct comparison of measured and approximated postural forces is shown in Figure 7: In Figure $7 A$ we have a measured

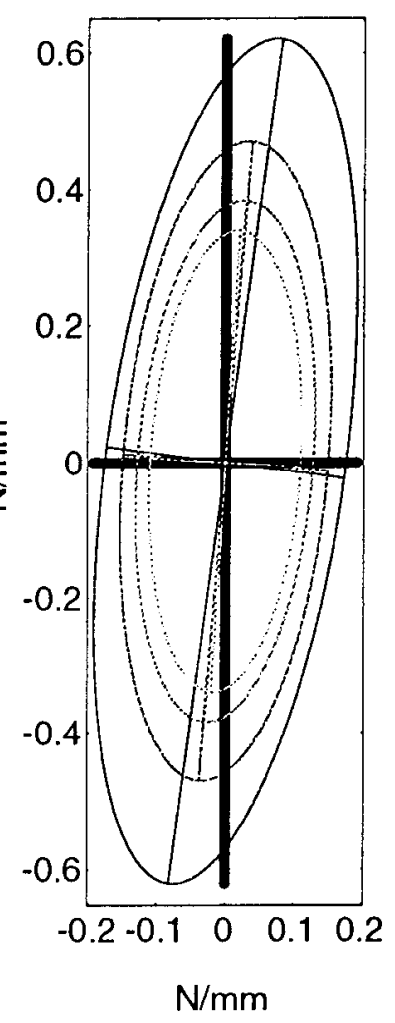

Figure 5. A graphical representation of the stiffness matrices fitted to the force-displacement data for two configurations of the arm at various distances from equilibrium (subject 2). A matrix was fitted to the measured restoring forces for a perturbation that displaced the hand by $0-1,1-2,2-3$, and $3-4 \mathrm{~cm}$. The ellipses are drawn progressively lighter in color as the displacement gets farther from equilibrium. Stiffness is largest for the smallest perturbation (0-1 $\mathrm{cm}$ from equilibrium). Stiffness becomes progressively smaller as the perturbation gets larger. $A$, The arm is in the "left" position. $B$, The arm is in the "right" position.

field of subject 2 at the left position of the arm, and in Figure $7 \mathrm{~B}$, the corresponding approximated field as produced by 25 basis fields.

From the fitted basis fields, it was possible to reconstruct the behavior of the postural force field at regular intervals around equilibrium. This is shown in Figure 8. Consider the restoring forces developed when the hand is $40 \mathrm{~mm}$ from equilibrium, that is, the forces at any point along the circumference of the circle that defines the periphery of the field. In general, these forces are not directed toward the equilibrium position. Furthermore, these forces have magnitudes that systematically change as the hand's positions with respect to the equilibrium position change. Now consider the consequences of the hy-

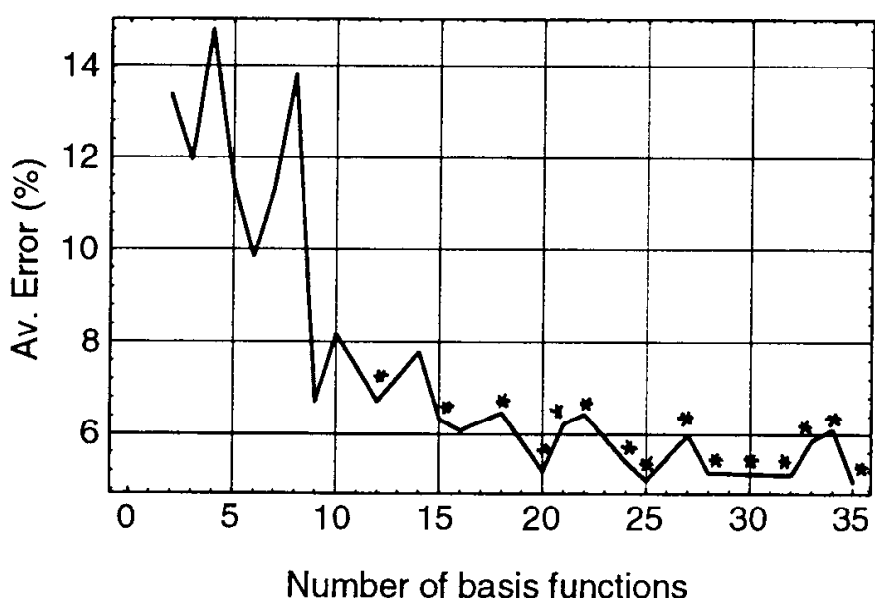

Figure 6 . The average error in fitting basis fields to the measured data of Figure $4 A$. The approximated data sets for which a statistically consistent distribution with the measured data was found (based on the Kolmogorov-Smirnov criterion) are marked by asterisks. 
Table 1. Basis function centers and coefficients for approximating the postural field of subject 1 at the "left" and "right" configurations of the arm

\begin{tabular}{|c|c|c|}
\hline \multirow[b]{2}{*}{$\begin{array}{l}\text { Basis centers } x_{i} \\
(\mathrm{~mm}, \mathrm{~mm})\end{array}$} & \multicolumn{2}{|c|}{ Basis coefficients $c_{i}$} \\
\hline & $\begin{array}{l}\text { Left position } \\
\text { (N) }\end{array}$ & $\begin{array}{l}\text { Right position } \\
\text { (N) }\end{array}$ \\
\hline$(-48,-40)$ & 201.71 & 391.34 \\
\hline$(-48,-20)$ & -44.82 & -343.83 \\
\hline$(-48,0)$ & 260.13 & 455.70 \\
\hline$(-48,20)$ & -44.52 & -468.73 \\
\hline$(-48,40)$ & 142.21 & 205.12 \\
\hline$(-24,-40)$ & -109.27 & -375.86 \\
\hline$(-24,-20)$ & 183.87 & 599.98 \\
\hline$(-24,0)$ & -179.29 & -477.02 \\
\hline$(-24,20)$ & 268.15 & 624.10 \\
\hline$(-24,40)$ & -207.15 & -394.97 \\
\hline$(0,-40)$ & 229.81 & 576.45 \\
\hline$(0,-20)$ & -107.45 & -575.51 \\
\hline$(0,0)$ & 406.31 & 717.27 \\
\hline$(0,20)$ & -236.72 & -545.80 \\
\hline$(0,40)$ & 371.97 & 454.23 \\
\hline$(24,-40)$ & -236.34 & -626.41 \\
\hline$(24,-20)$ & 352.01 & 785.17 \\
\hline$(24,0)$ & -386.71 & -652.26 \\
\hline$(24,20)$ & 558.47 & 760.71 \\
\hline$(24,40)$ & -515.93 & -477.59 \\
\hline$(48,-40)$ & 310.16 & 509.90 \\
\hline$(48,-20)$ & -201.80 & -687.34 \\
\hline$(48,0)$ & 506.28 & 668.80 \\
\hline$(48,20)$ & 430.18 & -532.15 \\
\hline$(48,40)$ & 472.99 & 478.38 \\
\hline
\end{tabular}

pothesis that a movement is generated via a program that somehow shifts the postural equilibrium position of the hand toward a target. In our case, the hand made reaching movements starting from points along the periphery of this field to a target at its center. In those trials where the hand was blocked from moving toward the target, the evoked forces should have the same relationship with respect to the target direction as the restoring forces do to the equilibrium position in Figure 8. This means that the evoked forces should, in general, (1) not be directed toward the target, and (2) not be equal for equal-distance movements. Furthermore, since the postural forces vary as a function of equilibrium position of the hand (the change in the fields when comparing Figs. $4 A, B$ or $8 A, B$ ), the same relative target direction in the two areas of the work space should result in different evoked forces. In the following, we report on the measured evoked forces and then quantify the consistency of the data with the predictions of the hypothesis.

\section{Measured evoked forces}

Subjects were instructed to move the robot's handle from points on the circumference of a circle of $4 \mathrm{~cm}$ to a target at its center. Trajectories of some typical movements are shown in Figure 9. In these movements, the brakes were not active and the manipulandum could be freely moved. Average movement time for this subject was $311 \mathrm{msec}$ (mean time for all subject was 318 msec).

In Figure 10 we have the static forces measured as the subject
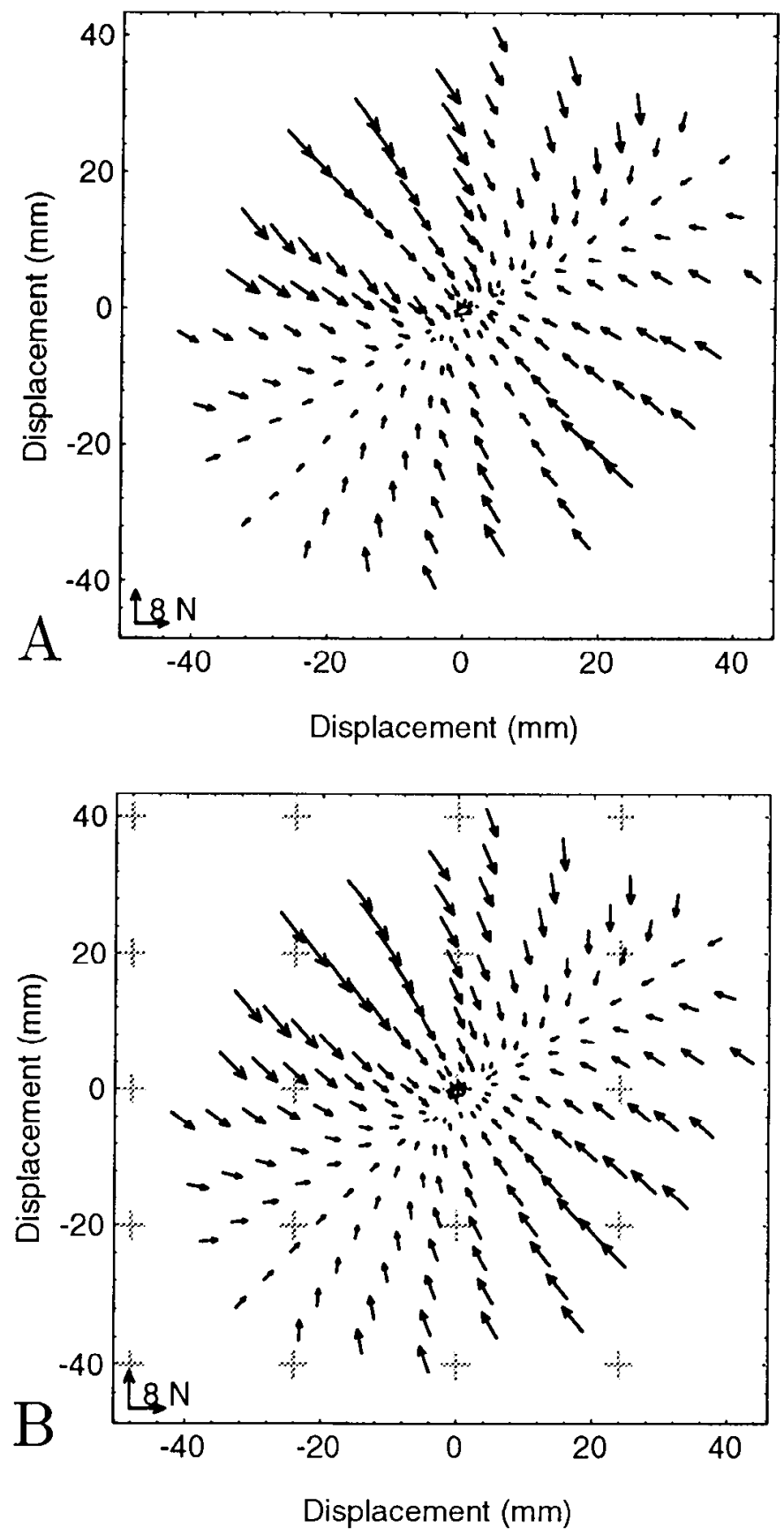

Figure 7. Measured postural forces and the forces produced through summation of a set of fitted basis fields in subject 2, "left" position. $A$, Measured postural field. Restoring forces are drawn at 1 sec intervals into the displacement perturbation. $B$. Approximated forces from the fitted basis fields. Centers of the basis fields are marked by shaded pluses. Coefficients of the basis fields are provided in Table 1. Average absolute error of the approximation: 0.48 and $0.53 \mathrm{~N}$ for $f_{x}$ and $f_{y}$, respectively. Correlation coefficient: $r^{2}=0.98$.

attempted to move the handle of the manipulandum to the target. The forces shown in this figure correspond to movement trials in which the display of the target coincided with the activation of the brakes and locking of the manipulandum for 300 msec. Approximately 170-200 msec after the display of the target, the subject began exerting a force on the manipulandum. The force grew in magnitude for $100 \mathrm{msec}$, and in most cases reached a plateau, at which point the brakes were released and 

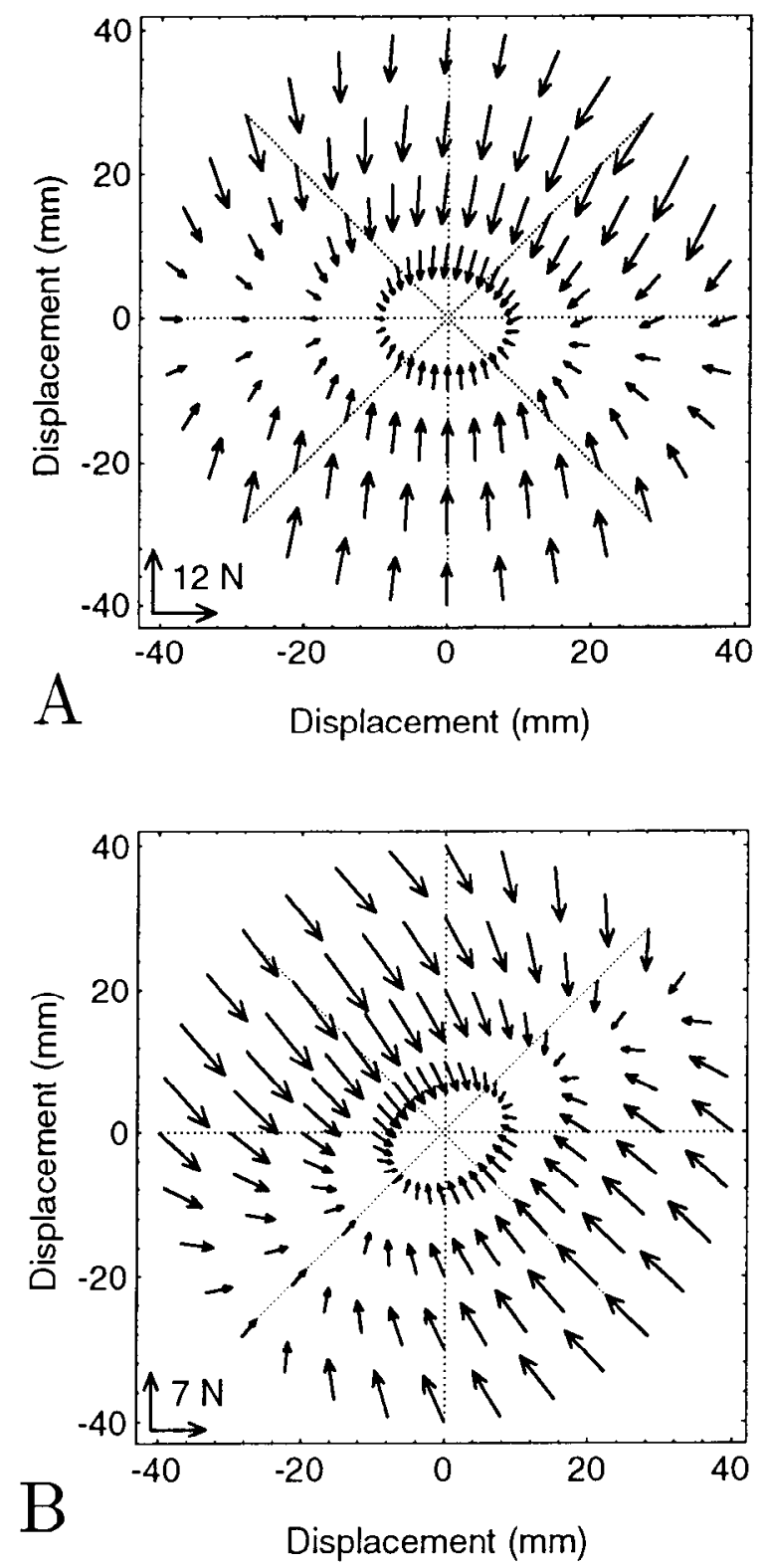

Figure 8. Restoring forces for subject 2 as computed from the basis fields, at two configurations of the arm. Forces are drawn at $10 \mathrm{~mm}$ intervals from equilibrium. $A$, The arm is in the "right" position. $B$, The arm is in the "leff" position.

the movement allowed to proceed. Once the brakcs wcrc rcleased, the movement lasted an average of $320 \mathrm{msec}$.

On the right side of Figure 10 we have indicated the direction of the target with respect to the starting position of the movement. Note that (1) the direction of the force vector is not necessarily equal to the direction of movement, (2) the force magnitude is different for different directions of movement although the target is at the same distance from the starting location, and (3) for some targets, for example, at $0^{\circ}$ and $180^{\circ}$, there is a rotation in the force vectors, while for other directions, for example, $90^{\circ}$ and $270^{\circ}$, there is only an increase in force magnitude without a change in force direction.

We represented the evoked forces produced by a subject for a given target by computing an average force vector during the period between 200 and $300 \mathrm{msec}$ after the target was presented. Figure 11 summarizes the average evoked forces for all move-

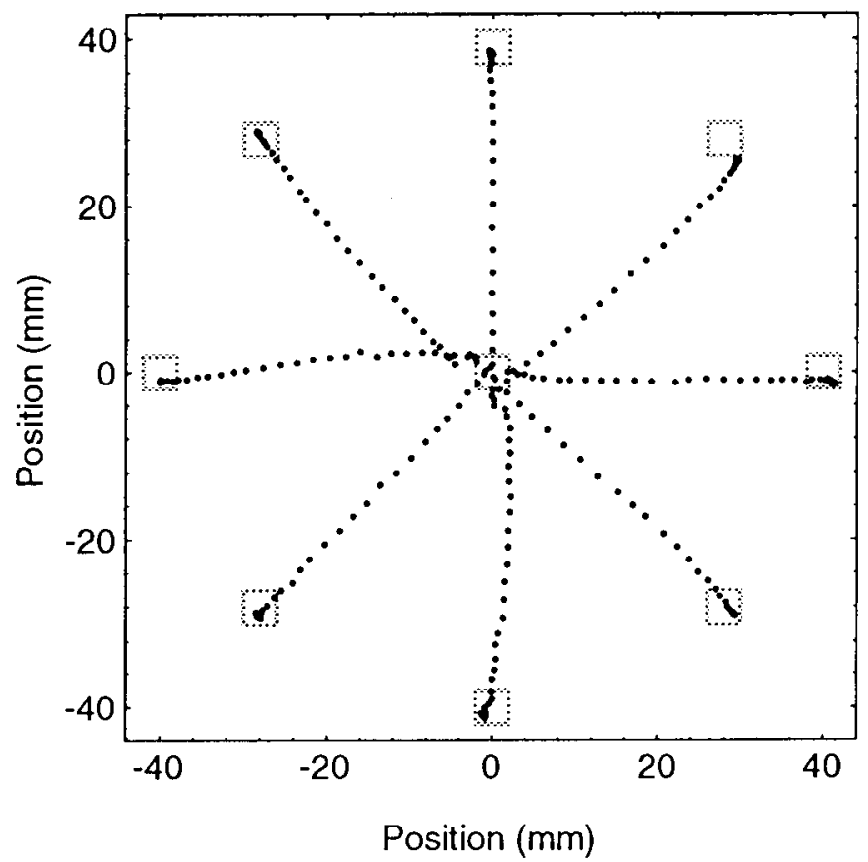

Figure 9. A set of trajectories for a subject moving the manipulandum from the periphery to the center target. The position dots are $10 \mathrm{msec}$ apart.

ment trials performed by a subject at the two configurations of the arm. We found that the forces generated over repeated movements to the same target were very consistent in both direction and magnitudc. In this figure, the shaded "wedges" are the mean \pm SD of the direction of the force vector generated by the subject for a movement toward a target at a given direction (this target direction is noted for each force vector). The length of each wedge is the mean magnitude of the evoked force, obtained by time averaging the force vectors during the period of application of the brakes. The solid line at the tip of each wedge is the SD of the force magnitude.

We found essentially the same pattern of evoked forces in the remaining six subjects, as illustrated by the data of three subjects shown in Figure 12. The differences between geometry of the arm (i.e., link lengths) among the subjects at the configurations tested contributed to the small differences that do exist in the pattern of evoked forces in this figure. As can be seen from these results, the evoked forces for movement toward a target were highly dependent on the configuration of the arm. In fact, there was a strong resemblance in the general shape of these evoked forces and the behavior of the postural forces in Figure 8.

\section{Comparing postural and evoked forces}

In Figure 13 we have plotted the direction of the restoring forces from the three separate measurements of the postural field: we calculated the direction of the restoring force from the postural field at $40 \mathrm{~mm}$ from equilibrium, that is, the forces at the periphery of the field in Figure 8. These force directions are plotted as a function of "target direction," where target direction refers to the direction of the equilibrium point. On the same figure we have plotted the mean \pm SD of the direction of the evoked forces.

If the movements were generated by shifting the equilibrium point to the center of the previously defined postural force field, then the evoked forces should be in the same direction as that 
Figure 10. Evoked forces measured in a subject for movements from a set of starting points on the circumference of a circle of $40 \mathrm{~mm}$ radius, to a target at its center. Direction of the target with respect to the start position is indicated on the far right. The target is shown to the subject at the point labeled $T / B$, which is also the time that the brakes are applied to the manipulandum. At the point labeled $R$, the brakes are released and the arm is allowed to move freely.

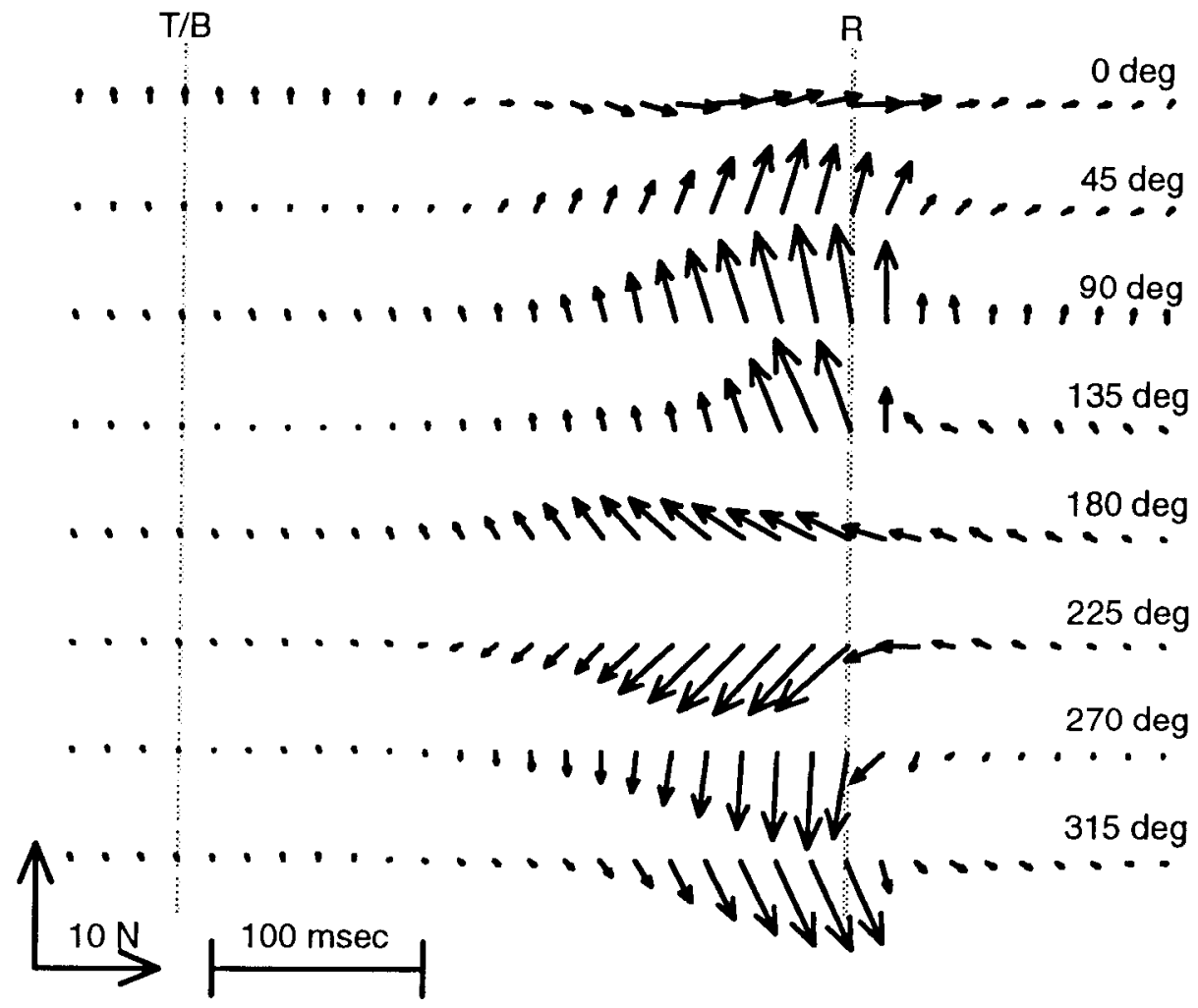

of the postural forces. Figure 13 shows that for both configurations of the arm, there is good agreement between the postural forces and the measured evoked forces: average absolute discrepancy in the measured and expected direction of evoked force in this subject was $7.8^{\circ}$ and $8.2^{\circ}$ for the right and left configurations, respectively. Average absolute discrepancy in all subjects tested was $6.1 \pm 1.9^{\circ}$ and $6.2 \pm 2.3^{\circ}$ for the right and left configurations, respectively.

From the postural field we can also describe the magnitude of the restoring forces for any point within a $40 \mathrm{~mm}$ radius of the equilibrium point. In Figure 14 we have plotted the magnitude of these forces from the measurements of the postural field. The same figure shows the relative magnitude of the evoked forces generated by the same subject during movements toward a target at the specified direction (the magnitudes were normalized with respect to the maximum force in the set). There is an impressive correspondence between the two forces in both configurations of the arm, consistent with the hypothesis that the subject initiated the movement by shifting his postural field toward the target. The average error between the magnitudes of the evoked and the postural forces for all directions across all subjects was $0.053 \pm 0.008$ relative force units (Fig. 14).

Figures 13 and 14 also demonstrate the repeatability of the three postural force fields measured in a typical subject. The three sets of dotted lines for direction and magnitude of the restoring force are very much in agreement with each other in these figures. This fact suggests that there is a high degree of stability in the shape and orientation of the postural fields.

\section{Reaching toward versus reaching away from the body}

The correspondence between the relative magnitudes of the postural and evoked forces can be further illustrated when we con- sider the evoked forces when a movement is made to a target that is toward the body as opposed to away from it. For example, consider the data in Figure 14B. It is an intriguing fact that when the hand was making a movement toward a target situated at $135^{\circ}$, the force generated was somewhat smaller than when the target was at $-45^{\circ}$; that is, for a movement directed at a target away from the body, the evoked forces were smaller than for a movement toward the body. Interestingly, this is precisely what would be expected if the movements were generated by shifting the equilibrium point: because of the nonlinear nature of the arm kinematics, the hand stiffness is expected to decrease when a subject is "pushing" away from the body, and to increase when a subject is "pulling" toward the body (McIntyre, 1990). As it can be seen in Figure $14 B$, the postural field produced smaller restoring forces when the equilibrium position was at $135^{\circ}$ versus $-45^{\circ}$. Accordingly, one would expect a smaller force if the equilibrium position had shifted to $135^{\circ}$ versus $-45^{\circ}$, which agrees with the results of the movement trials.

A deeper and more intuitive understanding of the nonlinear phenomenon associated with the postural force field can be gained by considering the change in the potential energy of the arm as the hand is displaced from equilibrium. Figure 15 illustrates this energy for the case where a subject's arm was in the left configuration. We calculated this energy function by using Equation 2 and effectively integrating the postural force field shown in Figure $8 B$. The equilibrium point is at the minimum of this energy function. When a perturbation moves the hand from equilibrium, the potential energy stored in the muscles of the arm increases (the spring-like muscles are stretched), and restoring forces result (force is the gradient of this energy function). Note that the energy stored in the arm is larger when the arm is pulled away versus the case where the arm is pushed toward the body. 

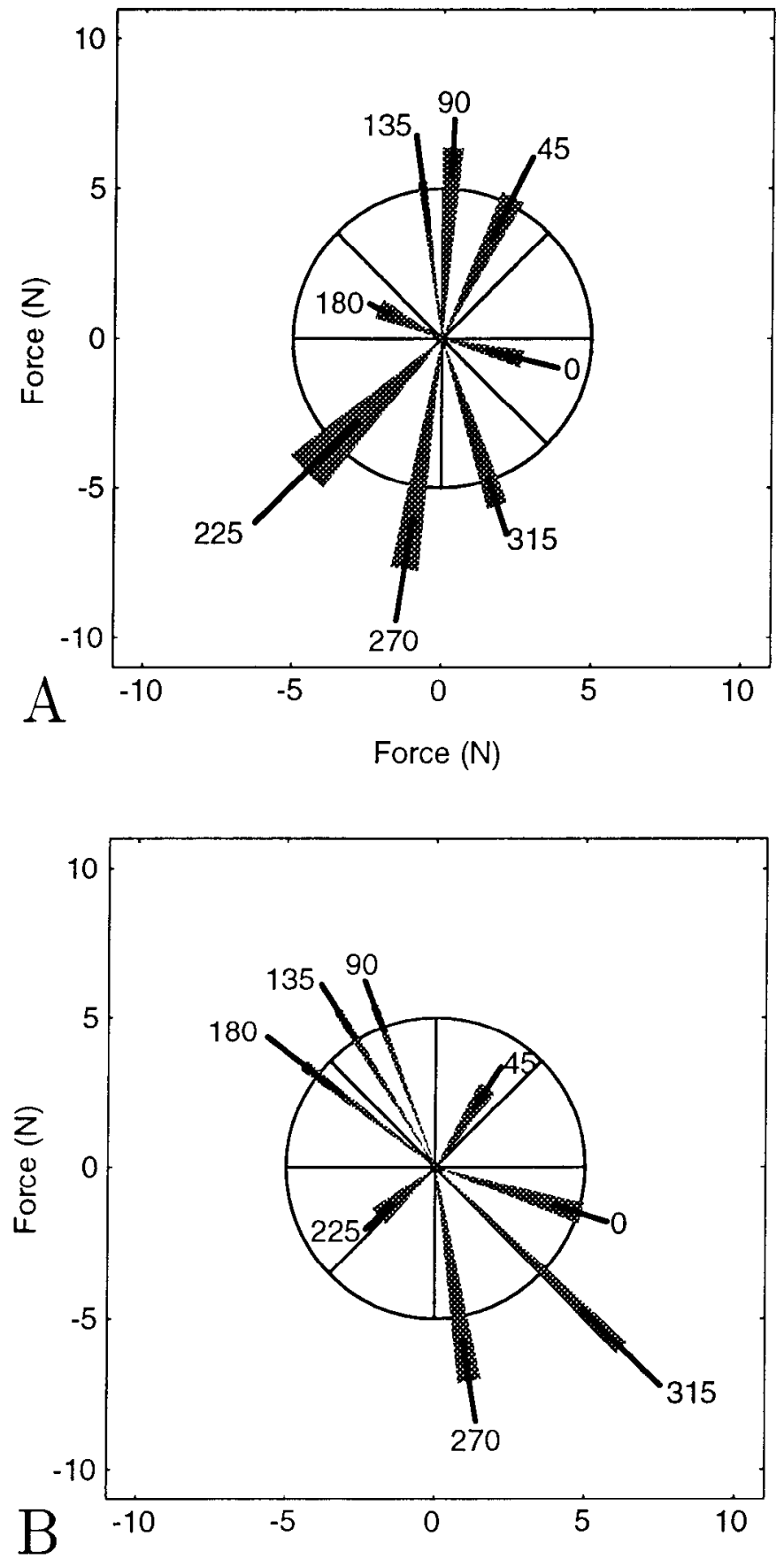

Force $(N)$

Figure 11. Evoked forces for eight movements trials at two arm positions for various target directions (data from subject 2). Evoked forces were averaged over the $100 \mathrm{msec}$ period before release of the brakes. The SD of intertrial force directions is indicated by the shaded wedges, while the SD of intertrial force magnitudes is shown by solid lines. A, The arm is in the "right" position. $B$, The arm is in the "left" position.

\section{Rotation in the evoked forces}

In the case of the movement task in the present study, when a target is presented to a subject, if the response is to shift the equilibrium point to the target, then since the hand is prevented from moving, it will effectively have a potential energy that is attracting it toward the target. This results in a force that we have shown to be strongly related to the evoked forces. Now,
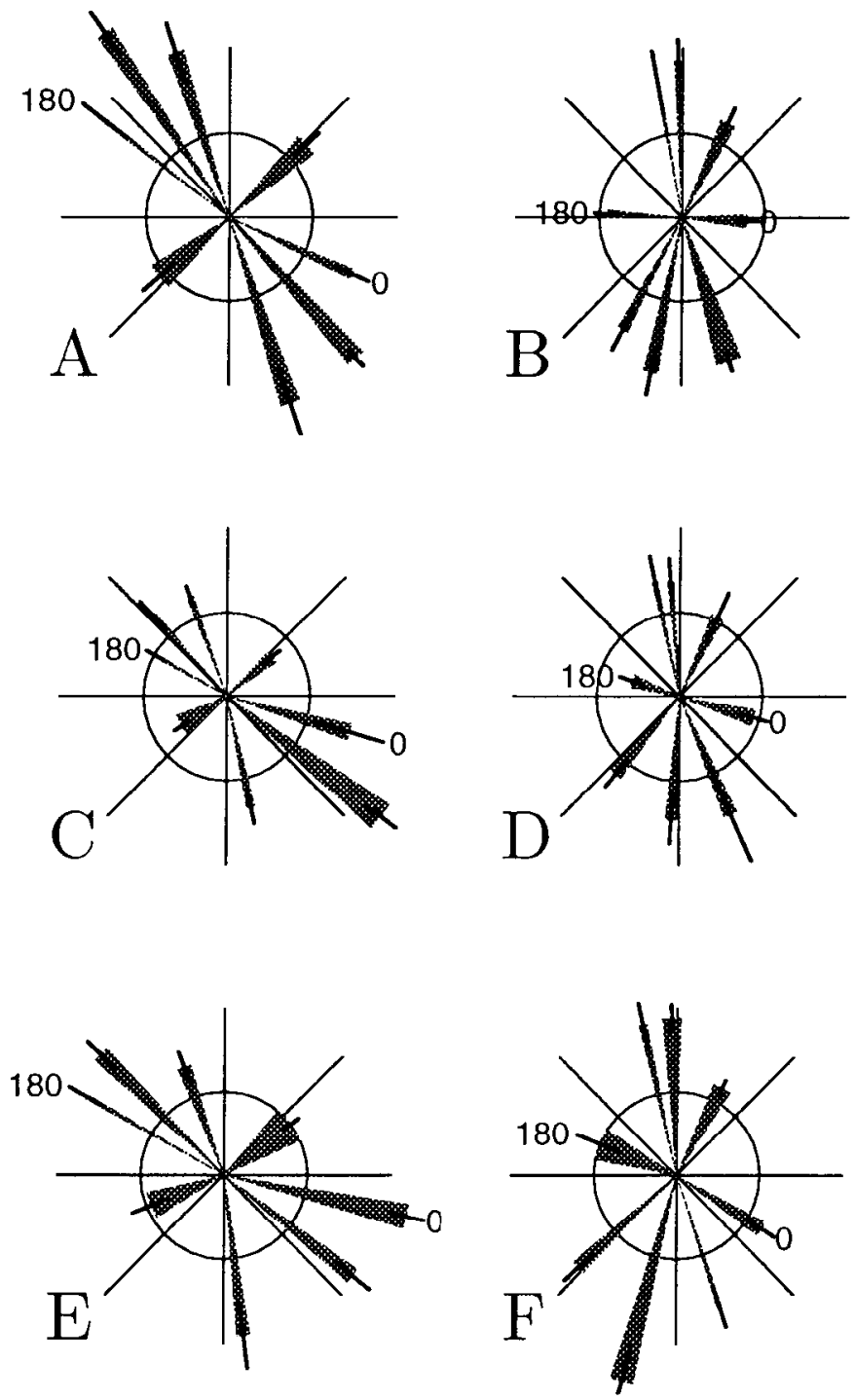

Figure 12. Mean and SD of the evoked force vector over eight movement trials at two arm positions for three subjects. The circle indicates $5 \mathrm{~N}$ of force. In the left column, the arm was in the left position, while in the right column, the arm was in the right position. Target directions are the same as in Figure 11. $A$ and $B$, subject $1 ; C$ and $D$, subject $3 ; E$ and $F$, subject 6 .

consider the direction of these forces that arise due to attraction to the "bottom of the bowl." In the region where the energy is high, the function is so steep that its gradient (the force) is always pointing toward the equilibrium point. On the other hand, where the energy is a shallow valley, small changes in position can lead to appreciable changes in the direction of the energy function's gradient. Both effects can be seen in the restoring forces of Figure $8 B$ : for displacements along the vicinity of $-135^{\circ}$ (corresponding to the shallow valley in Fig. 15), the direction of the restoring forces are extremely sensitive to the relative position of the hand to the equilibrium point. Comparatively, for displacement along the vicinity of $-45^{\circ}$ (corresponding to the steep region in Fig. 15), the direction of the restoring forces is far less sensitive.

Is this selective sensitivity to the relative position of the equilibrium point reflected in the evoked forces? If so, during a given movement trial, the evoked forces should vary more for some 

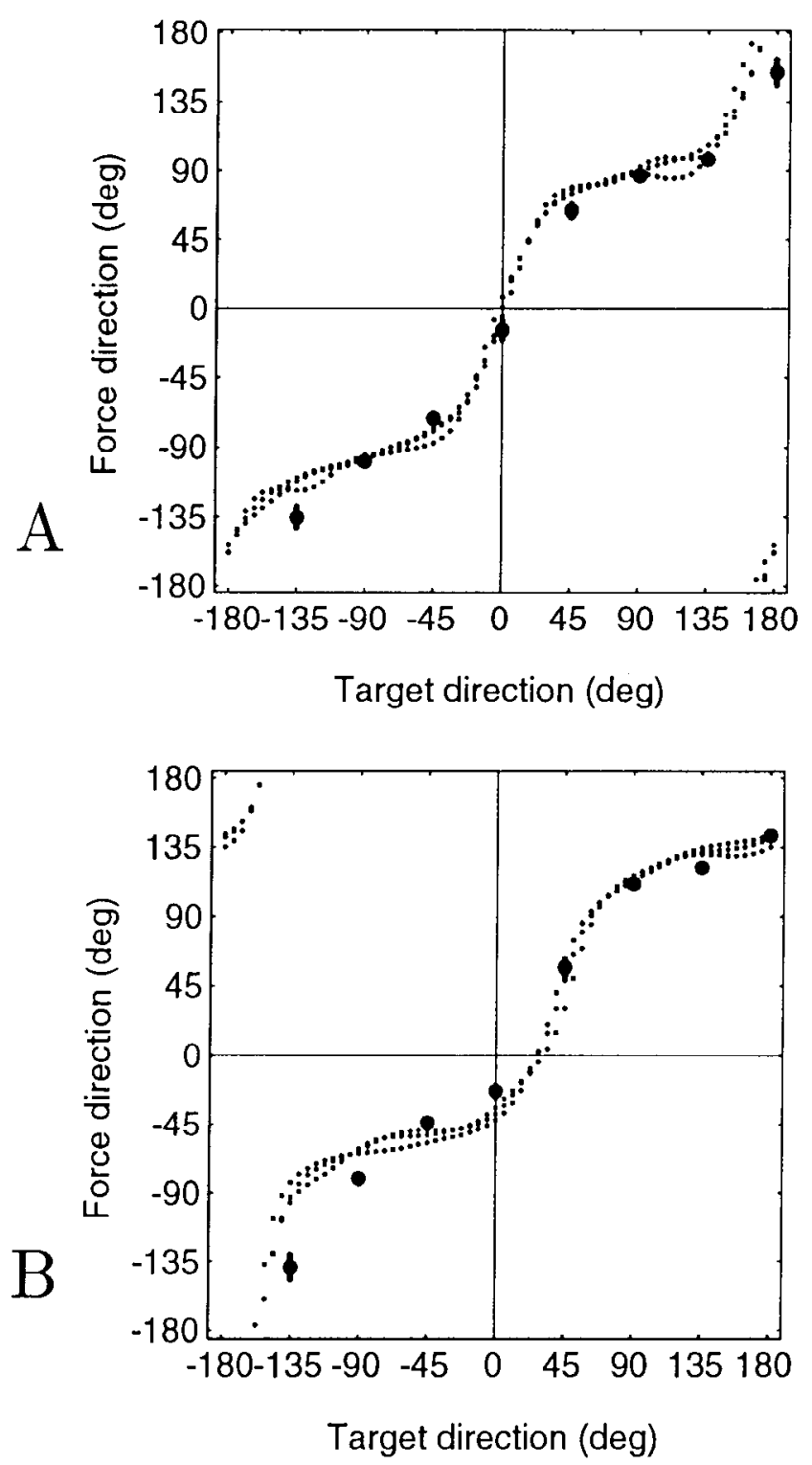

Figure 13. Relationship between direction of postural forces and evoked forces in subject 2. Direction of postural forces is drawn for each of the three measurements of postural field (dotted lines). Average direction of evoked force for a movement at a given direction is indicated by a solid circle. $A$, The arm is in the "right" position. Average absolute error in prediction: $7.8^{\circ} . B$, The arm is in the "left" position. Average absolute error in prediction: $8.2^{\circ}$.

targets than for others. In this scenario, the intratrial variability in the direction of the evoked forces would be a result of the sensitivity to the position of the equilibrium point as it moved to the target. Since this sensitivity is maximum for displacements along $45^{\circ}$ and $-135^{\circ}$ (when the arm is in the left configuration, as in Fig. $8 B$ ), we would expect the intratrial variability in the evoked forces also to be maximum for targets at $45^{\circ}$ and $-135^{\circ}$. In fact, the data are consistent with this prediction: in Figure 16 we have plotted the mean \pm SD of the intratrial variability in the direction of the evoked forces. For example, for a target at $45^{\circ}$, this subject's evoked forces showed a mean rotation of $11^{\circ}$. The amount of variability in the evoked forces is maximum for target at $45^{\circ}$ and $-135^{\circ}$, and minimum for targets at $-45^{\circ}$ and $135^{\circ}$. These are precisely the regions where the postural force field is most and least sensitive to the position
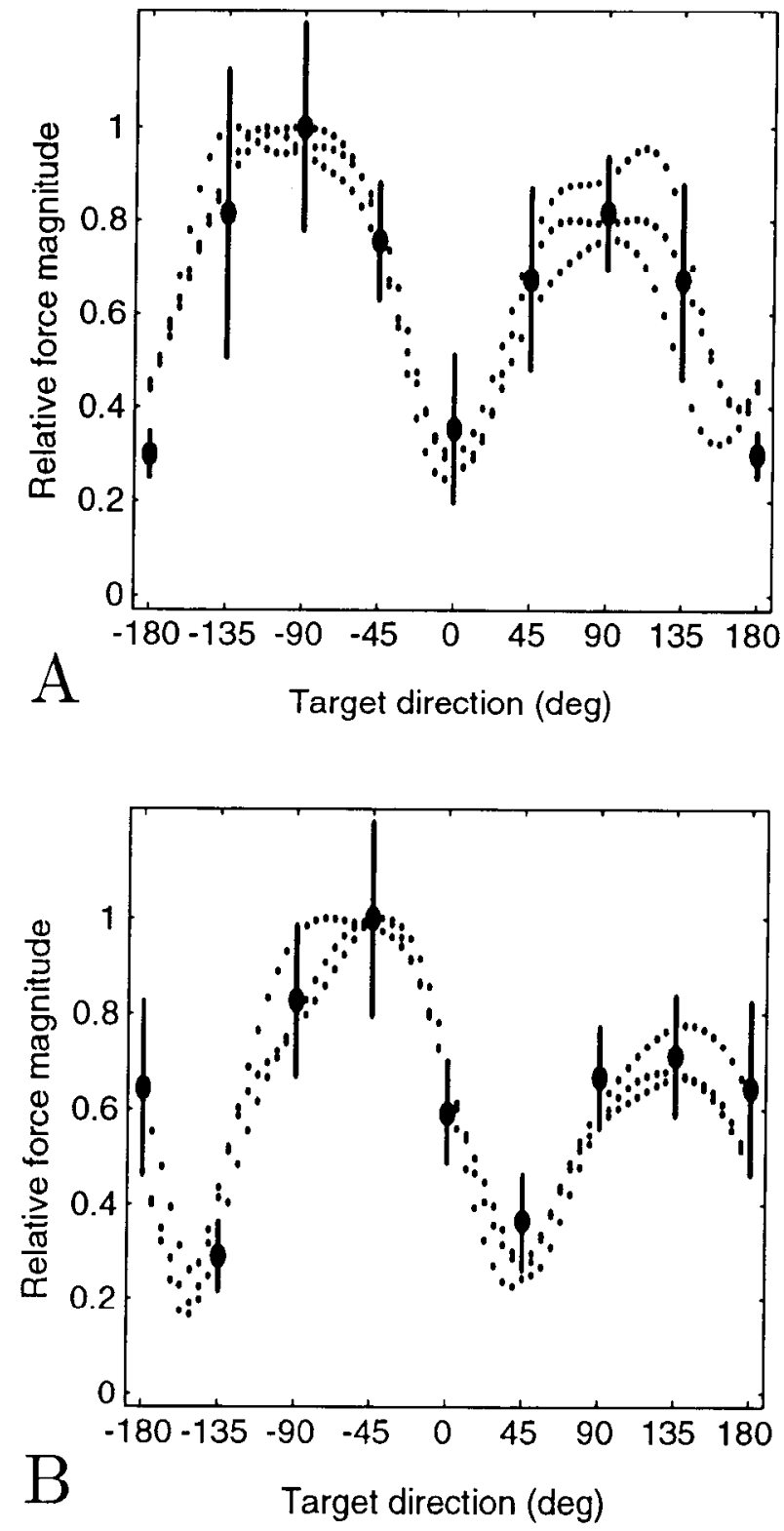

Figure 14. Relationship betwecn magnitude of postural forces and evoked forces in subject 2. Relative magnitude of postural forces (normalized with respect to itself) is drawn for each of the three measurements of postural field (dotted lines). Average magnitude of evoked force $( \pm \mathrm{SD})$ for a movement at a given direction is indicated by a solid circle and an error bar. $A$, The arm is in the "right" position. $B$, The arm is in the "left" position.

of the equilibrium point. In the case where the arm was in the right configuration, we found that the intratrial variability in the direction of the evoked forces was maximum for targets at $0^{\circ}$ and $180^{\circ}$ (see Fig. 10), which correlates with the behavior of the postural forces in Figure 8A. This pattern of intratrial force variations was present in all subjects.

\section{Discussion}

An equilibrium position refers to that configuration of the limb where the limb comes to rest in the absence of external disturbances or loads. The equilibrium trajectory hypothesis states that the CNS generates reaching movements by specifying a program that recruits muscles of the arm such that the equilibrium position of the limb is gradually shifted to the target. For 


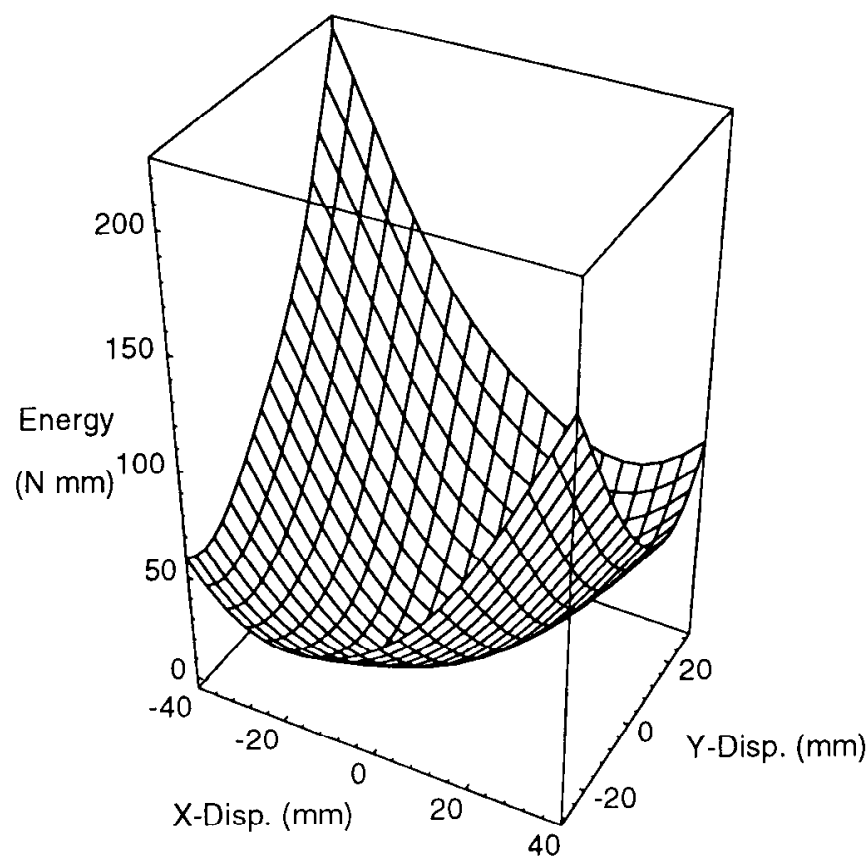

Figure 15. The computed potential energy gained in the arm of subject 1 as a function of displacement of the hand while maintaining posture at the "left" position. The $x$ - and $y$-axes refer to Figure 1. This potential function was computed from integration of the force field as constructed from the measured date (see Eq. 2). The steepest region of this energy function corresponds to the direction of maximum stiffness of the arm. The equilibrium point lics at the bottom of the "bowl."

multijoint movements, essentially the only evidence in support of this hypothesis has come from the work of Flash (1987), who showed that kinematic features of certain reaching movements can be accounted for by simulating a shift of the hand equilibrium position along a straight line from the start to the target position of the movement. Lack of other evidence has led some to suggest that, at least in its present form, the hypothesis may be untestable (cf. Jeannerod, 1988; Smith and Humphrey, 1991). The motivation of this study has been to take the first steps in quantifying some of the predictions of the hypothesis, and test whether these predictions are observed during reaching movements.

The static postural forces were quantified by slowly displacing the hand from equilibrium and measuring the restoring forces. These forces defined a field, which was a quantification of the program for posture. The center of the field where forces were zero was the equilibrium position of the limb, and the forces grew as the hand was displaced from equilibrium. We considered the postural forces for points that were at a distance of 40 $\mathrm{mm}$ from equilibrium. Based on the equilibrium point hypothesis, it was expected that when a movement is attempted from one of these points to a target at the center of the field, the static component of the force generated at the hand should match the characteristics (direction and relative magnitude) of the postural forces. Since the postural forces described a nonisotropic field that depended on the configuration of the arm, they provided a strong set of predictions regarding the static forces generated as a consequence of the program for motion. We estimated these static forces by instructing the subject to move the manipulandum to a target, while in random trials momentarily locking the system. We called the forces exerted on the manipulandum during this braking period "evoked forces."

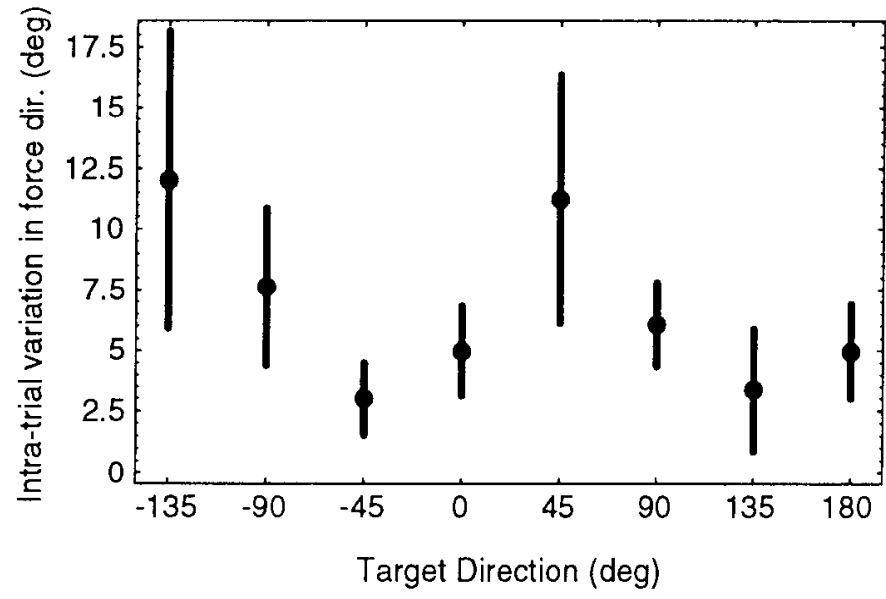

Figure 16. Intratrial variability in the direction of the evoked force vectors for various targets (data for subject 2). Maximum change in the direction of evoked forces was calculated for each movement trial. The means \pm SD of these changes over the eight repeated movements to the same targets are shown.

The results of this study show that, in agreement with the equilibrium point hypothesis, the pattern of evoked forces is remarkably similar to the pattern of the postural forces.

\section{Conditions for measuring a postural field and its repeatability}

The process of measuring the postural force field began with the manipulandum slowly displacing the arm from a resting position. During the displacement, the subjects were instructed to not voluntarily intervene. Our intentions, of course, were to minimize any voluntary contraction of the muscles during the process of perturbation and measure only the restoring forces due to the mechanical properties of stretched muscles and the action of the segmental reflexes.

This paradigm has been used by a number of researchers (Feldman, 1966, 1980; Crago et al., 1976; Vincken et al., 1983; Mussa-Ivaldi et al., 1985) and is perhaps the only method available for enforcing a constant "program state" in motor psychophysics. In the present study, there were a number of signs that indicated that the subjects kept the program for maintaining arm posture constant during the measurements of the postural forces. One indication was that the restoring force measured during a displacement was a smooth function. For example, in Figure $2 B$, note that both components of the force were monotonically and smoothly changing as a function of time. Clearly, onc sign that the subject may be actively reacting to the displacement would be for the forces to deviate from such a monotonic behavior.

A second indication regarding the success of the instruction not to intervene was the tendency of the arm to return to its original position after the completion of the perturbation. This is illustrated in Figure $2 A$, where after the torque motors were turned off, the arm/manipulandum system returned to within a few millimeters of its original position.

Perhaps the strongest indication of the success of this instruction was the degree of repeatability of the restoring forces when the arm was displaced at different times along the same direction. Since we measured the postural field three times in each subject, we had a way of measuring this repeatability. The highly correlated set of three dotted lines in Figures 13 and 14 give an indication of the remarkable stability of the fields over time. 
This repeatability was also observed in the data measured across subjects at a given configuration of the arm, as can be seen by comparing the fields measured from subjects 1 and 2 in Figures $4 B$ and $7 A$, respectively. Perhaps this is a manifestation of the strong influence that the geometry of the limb has on the pattern of torques that are produced at the joints as the hand is displaced from equilibrium. It should be pointed out, however, that at least in theory, the existence of double-joint muscles such as the biceps and triceps provides the required degree of freedom to affect the shape of this field substantially if the subject chose to do so (Hogan, 1980, 1985). Whether or not this degree of freedom is ever exercised through voluntary means remains an open question. It is, nevertheless, a curious fact that for the task of interacting with the manipulandum, neither we nor Mussa-Ivaldi et al. (1985) have observed a single case where the characteristics of the postural field differed substantially between subjects.

\section{Nonlinear approximation of the postural field}

The restoring forces due to perturbations from equilibrium were first measured by Mussa-Ivaldi et al. (1985). In their work, the hand was displaced by about $4 \mathrm{~mm}$ and restoring forces of about 1-2 N were measured. Since these perturbations were small, the restoring forces were modeled by a linear function of displacement. In the present study, due to the size of the displacements (about $40 \mathrm{~mm}$ ), we made no a priori assumption regarding the linearity of the restoring forces. In fact, several features of the postural field hinted at its nonlinearity with respect to displacement from equilibrium. (1) In Figure $4 A$, when the hand was displaced at $0^{\circ}$, the restoring forces were not opposite to the forces that were produced when the hand was displaced at $180^{\circ}$. This symmetry would be required if the force-displacement data could be well described by a linear model. (2) In Figure 5, stiffness of the force field was not constant (it decreased) as a function of the magnitude of displacement from equilibrium. This observation may be related to the results of Hoffer and Andreassen (1981), where it was noted that stiffness contribution of the spinal reflexes in the decerebrated cat decreased when the force produced by the muscle was in the medium to high range of the physiological range. Whether this is related to the declining stiffness observed in our data as the restoring forces increase remains an open question. (3) A displacement of the hand away from the body produced restoring forces that were larger in magnitude than when the hand was pushed toward the body (as illustrated by the forces in Fig. $4 B$ and magnitude of force traces in Fig. 14).

The postural forces were fitted to a nonlinear model that consisted of a set of predefined fields that were termed "basis fields." This is an extension of a technique used for represcnting nonlinear systems through approximation with a set of radial basis functions (Powell, 1987; Poggio and Girosi, 1990). We constructed each postural field through fitting the coefficients of the basis fields to the measured force-perturbation data. The basis fields themselves bore little resemblance to the measured data (e.g., compare Figs. $3 B, 4 A$ ), which was due to the nature of the potential function (Fig. $3 A$ ) used to arrive at the basis fields. This potential was a radial basis function. An indication that we succeeded in approximating the data while minimizing model complexity is the fact that with only 25 basis fields (i.e., a sum of 25 weighted fields), it was possible to construct a force field that was statistically consistent with the measured data, which numbered in the hundreds of vectors.

\section{Evoked forces}

The task was to move the handle of the manipulandum to a target. In randomly selected trials, the presentation of the target coincided with the locking of the manipulandum. Subjects exerted forces against the manipulandum for approximately 120 msec before the system was unlocked and the movement allowed to proceed (Fig. 10). The fact that these forces were remarkably repeatable in both direction and magnitude over several movement trials to a given target (Fig. 11) was an encouraging sign that a consistent criterion was being used to program these movements. The regularity in the pattern of forces in comparison to other targets (Fig. 11), and their repeatability across subjects (Fig. 12) were further evidence in favor of a common method used by the subjects to react to a target.

We observed a remarkable similarity when the evoked forces were compared to the forces produced during maintenance of posture at the target position. The direction and relative magnitude of these evoked forces were in agreement with the hypothesis that when a target is made visible, the subjects initiated a motor program that moved the equilibrium position of the hand to the target (Figs. 13, 14). A subtle feature of the evoked forces, namely, that of pulling harder when moving closer to the body than pushing when moving away from the body, was shown to be consistent when viewed in the framework of the potential energy of the arm when the equilibrium position of the hand is shifted to the target. Analysis of the time history of the evoked force data showed that in reaction to some targets, there were fluctuations in the direction of the evoked force vectors. This fact was illustrated in Figure 10 (arm in "right" configuration) for targets at $0^{\circ}$ and $180^{\circ}$, and as a whole for all directions in Figure 16 ( $\mathrm{arm}$ in "left" configuration). Interestingly, these fluctuations were prevalent for only those targets that were along the least stiff axis of the postural field, and correlated with the observation that the direction of the restoring forces was most sensitive to the position of the equilibrium if the hand was positioned at a point along the least stiff axis of the postural field.

In this analysis, we have compared evoked forces with postural forces when the equilibrium position was at the target of the movement. However, in single-joint movements, it has been shown that a transition of the equilibrium point does not happen instantaneously but follows a trajectory from the start to the final position (Bizzi et al., 1982). Based on the length of an unperturbed movement, the period of holding the limb before releasing it is approximately one-third of the total movement time, making it unlikely that the equilibrium point will have reached the target. Unfortunately, whether or not the motor program observed in the present study specified a straight line trajectory of equilibrium points rather than a jump in the equilibrium position is a question that this level of analysis is unable to answer. Because of the small size of the movement, an equilibrium point positioned at one-third of the way between the start and the target position (along a straight line) would not give a force significantly different in direction than when the equilibrium position was at the target: the orientation of the postural field, as estimated from the direction of the principal eigenvector of the stiffness ellipse, rotates at most $2.7^{\circ}$ from the start position to the target of any movement. An indication of this is apparent by comparing the stiffness ellipses when the hand is $1-2$ versus $3-4 \mathrm{~cm}$ from equilibrium in Figure 5 . There is little change in the orientation (direction of maximum stiff- 
F

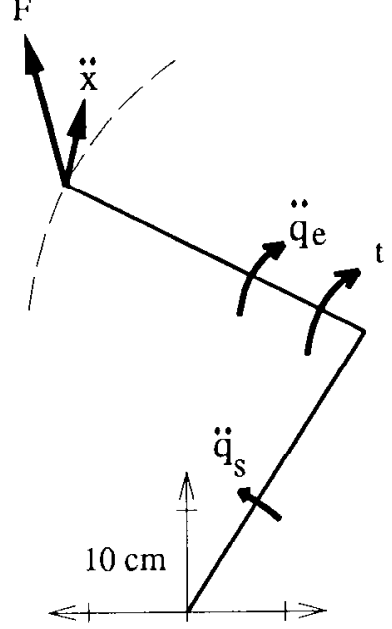

Figure 17. An example of how torque produced in one joint causes acceleration in both joints and produces a force at the hand that is not aligned with the direction of acceleration. The configuration of the arm is at the "left" target. We assume that muscles acting on the elbow produced an extension torque. This results in acceleration in both joints and a force at the hand that is not tangent to the arc of rotation about the elbow. $t$, torque; $F$, force; $\ddot{q}_{s}$ and $\ddot{q}_{e}$, shoulder and elbow accelerations, respectively; $\ddot{\mathbf{x}}$, the acceleration vector of the hand.

ness) of the ellipses. Any change in the orientation of the postural field due to movement of an equilibrium point is likely to be within $1 \mathrm{SD}$ of the direction of the evoked forces for a single movement trial.

\section{An alternate hypothesis: compensating for inertia}

If the effects of the postural force field were not known, then it might be difficult to explain the above-mentioned properties of the evoked forces. One possible scenario, however, might be that the evoked forces were due to a program that was attempting to accelerate the arm toward the target (Ghez et al., 1990). If so, then the underlying program would have to take into account the fact that producing torque on one joint may lead to acceleration in not only the affected joint, but also neighboring joints as well (Hollerbach and Flash, 1982).

There is some evidence that indicates that one aspect of motor planning may be to take these interaction torques explicitly into account; for example, Aoki (1991) has shown that for voluntary movements in the wrist, activity in the elbow muscles may be such that they minimize the acceleration that is caused on the elbow joint when the wrist muscles contract. This causality can be shown in the framework of arm movements as well: assume that in the arm schematic of Figure 17, the "hand" is grabbing the manipulandum (not shown) and the brakes are activated so that there is no motion in the system. Consider the behavior of the arm when the elbow extensors become active. The torque at the elbow leads to a force at the hand. Note that this force is not tangent to the arc that results from rotation of the elbow joint. If at this moment the brakes were turned off and the arm allowed to move, both elbow and shoulder joints would accelerate, resulting in the hand accelerating along the vector shown. This acceleration vector is a function of the inertia of the limb. Parameter values used for this simulation were from cadaver information (Diffrient et al., 1978) and have been shown to be adequate in other studies (Russell, 1990). The transformation from torques to acceleration can be derived from Newtonian
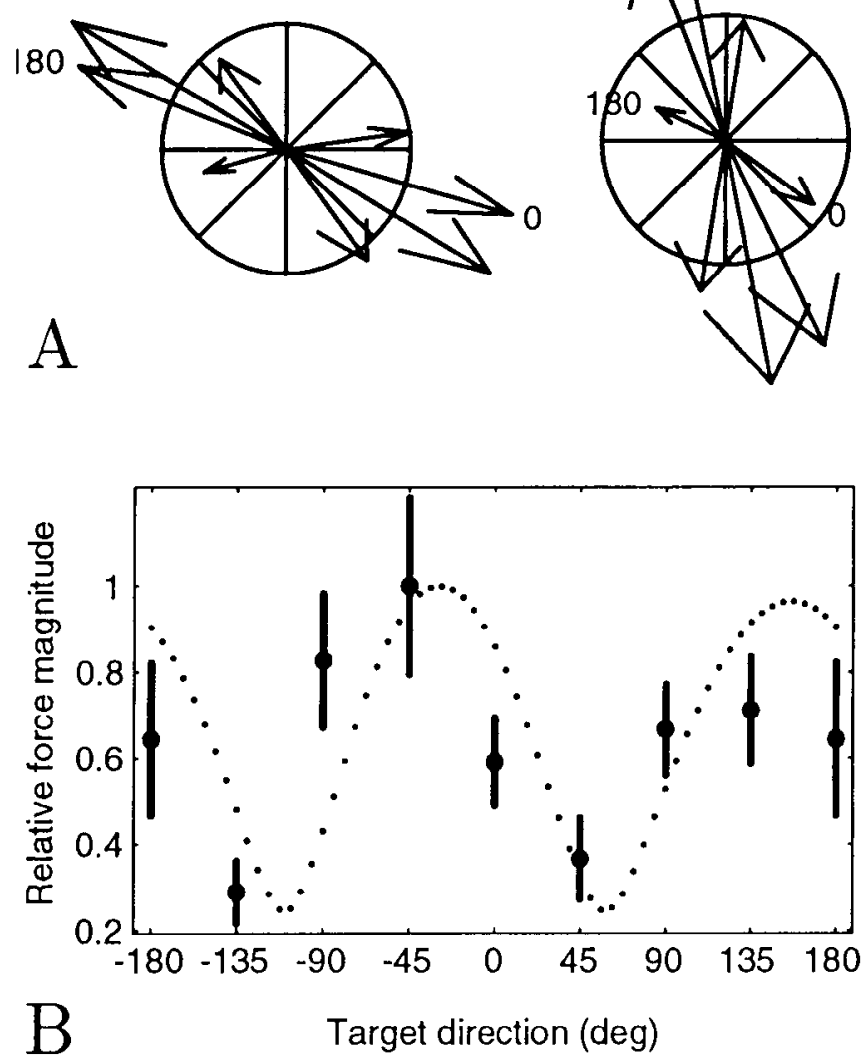

Figure 18. Expected evoked forces if the CNS had programmed muscle activities so to accelerate the hand by a unit vector pointing toward a target (simulations based on the kinematics of subject 2). $A$, Forces required to accelerate the arm toward a target for the "left" $(l e f t)$ and "right" configurations of the arm (right). Predicted forces for eight targets evenly spaced at $45^{\circ}$ intervals are shown. Forces for targets at $0^{\circ}$ and $180^{\circ}$ are identified. $B$, Predicted (dotted line) and measured evoked force magnitudes versus target direction when the hand was in the "left" position

mechanics. The transformation from torques to forces can be derived from the principle of virtual work (cf. Craig, 1986).

From this example, we can see that it is possible that the distortions observed in the evoked forces may be due to a strategy that attempted to accelerate the hand toward a target. In Figure $18 \mathrm{~A}$, we have calculated the expected evoked forces from this hypothesis; these predicted forces are based on the kinematics of the subject whose measured evoked forces were shown in Figure 10. The inertia of the arm is such that in order to accelerate the hand toward a target that is aligned with the forearm, one needs to produce much more force than is required when the goal is to accelerate the hand at a perpendicular direction.

The shape of the inertia tensor dictates that the predicted evoked forces will be oriented toward the elbow joint (compare with the kinematics of the forearm in Fig. 1) if the goal is to accelerate toward the target. In contrast, the measured evoked forces for this subject (Fig. 11) were aligned toward the shoulder joint. Furthermore, the predicted magnitudes of force based on this hypothesis do not agree very well with the relative magnitudes of evoked forces measured. This is shown in Figure $18 B$, where the dotted line is the predicted magnitude of forces that 


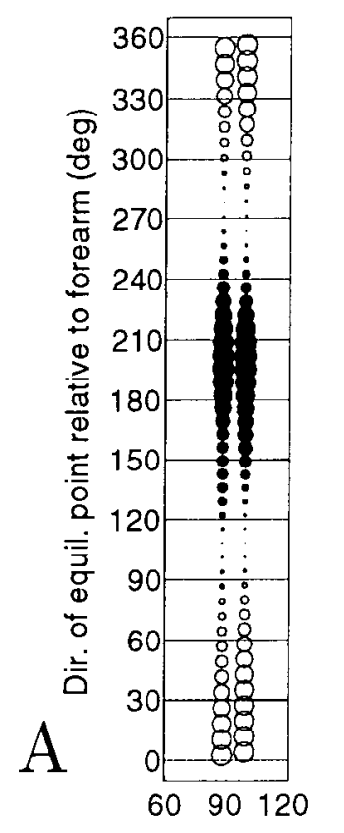

Elbow angle (deg)

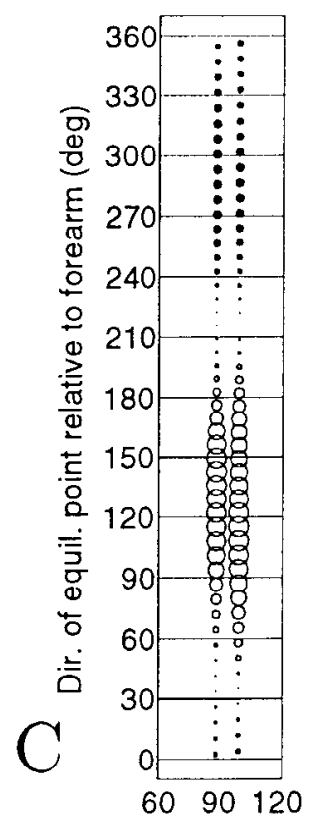

Elbow angle (deg)

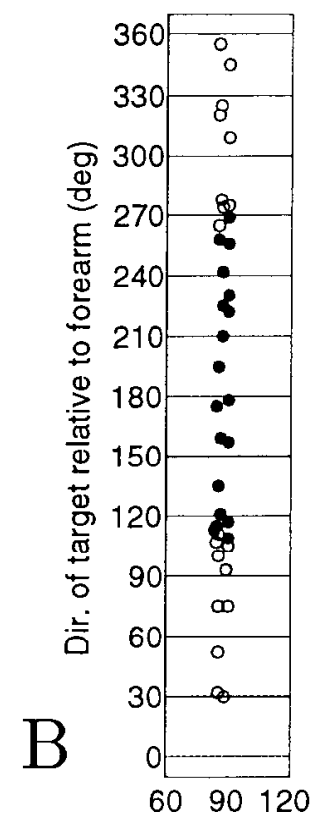

Elbow angle (deg)

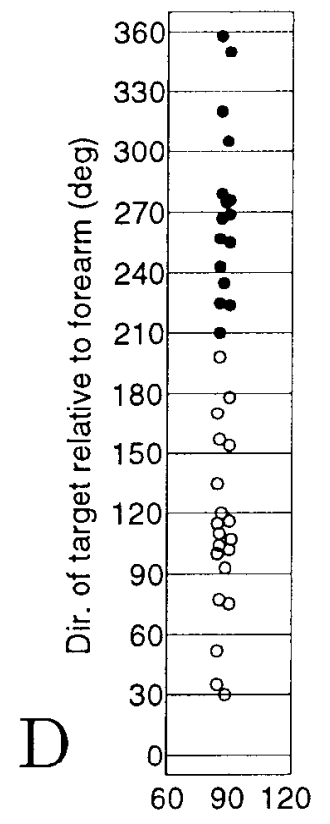

Elbow angle (deg)
Figure 19. Restoring torques produced at the shoulder and elbow joints of subject 1 in response to a displacement from equilibrium as compared to the "sign" of EMG on the arm muscles during initiation of reaching movements from subject 1 of Karst and Hasan (1991). $A$ and $C, \operatorname{Re}-$ storing torques at the shoulder and elbow from the postural force field at the two configurations of the arm. Open circles are flexion torques; solid circles are extension. The size of each circle is normalized to reflect the magnitude of the torque. The abscissa is the elbow joint angle at which the postural fields were measured. The ordinate is the direction of the equilibrium point as expressed in the coordinate system of Karst and Hasan (1991). $B$ and $D$, Sign of EMG (flexion or extension) of the shoulder and elbow muscles for initiation of reaching movements to various targets. Flexion is noted by open circles; extension, by solid circles. should have resulted if the goal was to accelerate the hand toward the target.

These simulations indicate that the inverse dynamics hypothesis does not fare as well as the equilibrium point model with the measured data. Interestingly, recent results from Karst and Hasan (1991) seem to confirm the notion that forces generated during initiation of an unperturbed reaching movement are not as would be expected if the goal was to accelerate toward the target. This work and the question of whether the postural forces are related to forces generated during a free reaching movement are discussed next.

\section{Comparison to free reaching movements}

In this study we have documented the existence of a strong link between posture and movement. Our conclusions, however, are based on movement trials that altered the program for motion by preventing the movement (albeit for only $120 \mathrm{msec}$ or so of the movement), and it may be difficult to compare the evoked forces measured here to static forces that would have been generated in the absence of the movement blockage. Furthermore, since the movements were of relatively small magnitude $(4 \mathrm{~cm})$, they may not be a good representative of reaching movements in general. Nevertheless, it is possible to show that the postural forces measured in this study are in fact linked to forces generated in initiation of unperturbed reaching movements of much larger magnitude.

There are timing factors that suggest that the evoked forces represent the static component of an on-going program for motion. Several studies have evaluated the time it takes for proprioceptive feedback to make an adjustment in the motor program specified by the CNS. This kinesthetic reaction time has been estimated at 110-160 msec (Vince, 1948; Chernikoff and Taylor, 1952; Evarts and Vaughn, 1978), and it is believed that "the first $100 \mathrm{msec}$ of any movement should be under the exclusive control of central factors being unaffected by reafferent kinesthetic signals" (Jeannerod, 1991, p. 278). Therefore, based on timing factors, it may be said that the correspondence between the evoked forces and the postural forces is consistent with the hypothesis that the program for motion was a shift of the equilibrium toward the target. However, other evidence from unobstructed reaching movements may be provided that makes this link from another perspective, namely, that of muscle activations.

Hasan and Karst (Hasan and Karst, 1989; Karst and Hasan, 1991) have measured the "sign" of the EMG (flexion or extension lorque) acting on the shoulder and elbow joints as a completely relaxed arm, supported on a frictionless mechanism, makes movements of various amplitude in most parts of the reachable space. The sign of the EMG was interpreted by Karst and Hasan (1991) to represent the direction of the net torque produced by the muscles on the arm; for example, when the movement was initiated by an EMG burst in the elbow flexors but not extensors, it was assumed that the initiation torque was in the direction of flexion. Using this assumption, three hypotheses were tested: (1) Kinematic hypothesis - torques are in same direction as the rotation in the joint angles required to move the hand in a straight line to the target; (2) Direction of Force hypothesis-torques are such that the hand produces a force that points to the target; and (3) Inverse Dynamics hypothesis-torques are such that the hand accelerates toward the target. The measured data did not support any of these hy- 
potheses. Karst and Hasan (1991) did not consider the hypothesis of a shift of the equilibrium point because it was conjectured that since the arm was completely relaxed, there would be no relationship between postural stiffness and movement EMGs.

It should be noted that although Karst and Hasan (1991) have suggested that the predictions of the Direction of Force hypothesis are similar to those of the equilibrium point hypothesis (cf. Berkinblit et al., 1986), this is, of course, not the case becausc the postural field is in general anisotropic. In fact, Shadmehr (1991) has shown that the direction of torques reported in Hasan and Karst (1989) was as would be expected if the subject's arm had a postural stiffness that was typical in its orientation to the measurements made by Mussa-Ivaldi et al. (1985), and the movement was initiated by a shift of the postural field defined by this stiffness toward the target. In order to illustrate this point further, in what follows we have compared the results of Karst and Hasan (1991) regarding the sign of EMG during free movements, and direction of torques produced by our postural fields during displacement from equilibrium. We ask whether the sign of EMG reported by Karst and Hasan (1991) for a free movement is consistent with the hypothesis that the movement was generated by a shift of that subject's postural force field toward the target. This comparison is possible because many of the reported data points in Karst and Hasan (1991) were from joint configurations that corresponded to our two positions where tests were performed.

In Figure 19, $A$ and $C$, we have plotted the direction and relative magnitude of the restoring torques at the shoulder and elbow joints, respectively, when the hand of subject 1 is displaced from equilibrium (each of these panels shows the restoring torques at both of the arm configurations tested). The torques were computed from the force field of Figure 4. In Figure $19, B$ and $D$, we have the sign of EMG (flexion or extension) as observed by Karst and Hasan (1991) from the shoulder and elbow joints, respectively, for movements to various target directions. The coordinate system used to plot these torques and EMGs is as used by Karst and Hasan (1991): in Figure 19, $A$ and $C$, the abscissa is the angle of the elbow joint at which the postural fields were measured, while in $B$ and $D$, the abscissa is the angle of the elbow joint from which movements were made. In Figure 19, $A$ and $C$, the ordinate is the direction of the equilibrium point with respect to the orientation of the forearm, while in $B$ and $D$, the ordinate is the target direction with respect to the orientation of the forearm. Target (or equilibrium point) direction with respect to the orientation of the forearm is the difference between the target direction as expressed in an extrinsic coordinate system and the sum of the elbow and shoulder joint angles (see Fig. 1 of Karst and Hasan, 1991).

In comparing Figure $19, A$ to $B$ and $C$ to $D$, it is apparent that the transition between a flexion and an extension torque in the postural data occurs at the same direction of displacement from equilibrium point as the transition between the flexion and extension EMGs for movement targets. It is a remarkable fact that although these crucial transition points could not be reproduced by the three hypotheses tested by Karst and Hasan (1991), they are in clear agreement with the postural forces measured in our study: initiation torques for free reaching movements are in the same direction as restoring torques that result when the hand is displaced from an equilibrium point that has been shifted toward the movement's target. This provides further evidence that the close relationship between evoked forces measured in our movements and the postural field may in fact be a measurable expression of the neural program for motion.

Interestingly, Karst and Hasan (1991) have reported that the sign of the EMGs, as represented in this format, does not vary as a function of movement distance, nor does it vary when the arm's dynamics have been changed because of a significant load. This would suggest that the notions of equilibrium point control may be valid for a very large repertoire of movements, which, for example, would predict that the evoked forces should remain invariant in direction and only be scaled as the target of the movement is put at larger distances. This question, along with that of the effect of an added load, remains to be answered in further studies.

The results of our study may be relevant to the recent findings that the "preferred directions" of the cortical neurons do not remain fixed as represented in extrinsic coordinates, but instead follow the change in the shoulder angle. This was found to be true for neurons in the premotor cortex (Caminiti et al., 1990a) as well as the primary motor cortex (Caminiti et al., 1990b). The rotation of the preferred directions as a function of the shoulder angle is intriguing because the postural force field also rotates with the shoulder joint. Whether or not there is a relationship between the neural code in the cortex and the act of moving an equilibrium point for generating a movement remains to be seen. Our findings may also be relevant to the analysis of trajectories of reaching movements in functionally deafferented patients (Ghez et al., 1990). The conclusions of that study regarding the ability of the patients to compensate for the inertia of their arm was partly based on the assumption that a reaching movement to a target would initiate with a force pointing to the target, which is not supported by the data in this study.

In conclusion, because of the nature of restoring forces developed during maintenance of multijoint posture, the equilibrium trajectory hypothesis makes strong predictions regarding the direction, magnitude, and variance of static forces produced at the hand during initiation of reaching movements. Our measurements of these forces are consistent with the predictions of the hypothesis, asserting the significant role of posture in generation of multijoint movements.

\section{References}

Aoki $F$ (1991) Activity patterns of upper arm muscles in relation to direction of rapid wrist movements in man. Exp Brain Res 83:679-682. Berkinblit MB, Gelfand IM, Feldman AG (1986) Model of the control of the movements of a multijoint limb. Biophysics 31:142-153.

Bizzi E, Polit A, Morasso P (1976) Mechanisms underlying achievement of final head position. J Neurophysiol 39:435-444.

Bizzi E, Dev P, Morasso P, Polit A (1978) Effect of load disturbances during centrally initiated movements. J Neurophysiol 41:542-556.

Bizzi E, Accornero N, Chapple W, Hogan N (1982) Arm trajectory formation in monkeys. Fxp Brain Res 46:139-143.

Bizzi E, Accornero N, Chapple W, Hogan N (1984) Posture control and trajectory formation during arm movement. J Neurosci 4:2738-2745.

Caminiti R, Johnson PB, Urbano A (1990a) Making arm movements within different parts of space: dynamic aspects in the primate motor cortex. J Neurosci 10:2039-2058.

Caminiti R, Johnson PB, Burnod Y, Galli C, Ferraina S (1990b) Shift of preferred directions of premotor cortical cells with arm movements performed across the workspace. Exp Brain Res 83:228-232.

Chernikoff R, Taylor F (1952) Reaction time to kinesthetic stimulation resulting from sudden arm displacement. J Exp Psychol 43:1-8.

Crago PE, Houk JC, Hasan Z (1976) Regulatory actions of human stretch reflex. J Neurophysiol 39:5-19. 
Craig JJ (1986) Introduction to robotics. Reading, MA: AddisonWesley.

Diffrient N, Tilley AR, Bardagjy JC (1978) Humanscale. Cambridge, MA: MIT Press.

Evarts EV, Vaughn WJ (1978) Intended arm movements in response to externally produced arm displacements in man. Prog Clin Neurophysiol $4: 178-192$.

Feldman AG (1966) Functional tuning of the nervous system with control of movement or maintenance of posture-II. Controllable parameters of the muscles. Biophysics 11:565-578.

Feldman AG (1980) Superposition of motor programs-I. Rhythmic forearm movements in man. Neuroscience 5:81-90.

Flash T (1987) The control of hand equilibrium trajectories in multijoint arm movements. Biol Cybern 57:257-274.

Ghcz C, Gordon J, Ghilardi MF, Christakos CN, Cooper SE (1990) Roles of proprioceptive input in the programming of arm trajectories. Cold Spring Harbor Symp Quant Biol 55:837-847.

Hasan Z (1991) Moving a human or robot arm with many degrees of freedom: issues and ideas. In: Lectures in the sciences of complexity III (Nadel L, Stein DL, eds). Redwood City: Addison-Wesley.

Hasan Z, Karst GM (1989) Muscle activity for initiation of planar, two-joint arm movements in different directions. Exp Brain Res 76: 651-655.

Hoel PG, Port SC, Stone CJ (1971) Introduction to statistical theory. Boston: Houghton Mifflin.

Hoffer JA, Andreassen S (1981) Regulation of soleus muscle stiffness in premammillary cats: intrinsic and reflex components. J Neurophysiol 45:267-285.

Hogan N (1980) Mechanical impedance control in assistive devices and manipulators. In: Proceedings of the 1980 Joint Automatic Control Conference, paper TA 10-B.

Hogan N (1985) The mechanics of multi-joint posture and movement control. Biol Cybern 52:315-331.

Hollerbach JM, Flash T (1982) Dynamic interactions between limb segments during planar arm movement. Biol Cybern 44:67-77.

Jeannerod M (1988) The neural and behavioural organization of goaldirected movements, pp 25-26. Oxford: Clarendon.

Jeannerod M (1991) The interaction of visual and proprioceptive cues in controlling reaching movements. In: Motor control: concepts and issues (Humphrey DR, Freund H-J, eds), pp 277-291. Chichester: Wiley.
Karst GM, Hasan Z (1991) Initiation rules for planar, two-joint arm movements: agonist selection for movements throughout the work snace. J Neurophysiol 66:1579-1593.

Kelso JAS, Holt KG (1980) Exploring a vibratory system analysis of human movement production. J Neurophysiol 43:1183-1196.

McIntyre J (1990) Utilizing elastic system properties for the control of posture and movement. PhD thesis, Massachusetts Institute of Technology.

Mussa-Ivaldi FA, Hogan N, Bizzi E (1985) Neural, mechanical, and geometric factors subserving arm posture. J Neurosci 5:2732-2743.

Mussa-Ivaldi FA, Bizzi E, Giszter SF (1991) Transforming plans into actions by tuning passive behavior: a field approximation approach. In: Proceedings of the IEEE International Symposium on Intelligent Control, pp 101-109. Arlington, VA: IEEE.

Nichols TR (1989) Coordination of muscular action in cat hindlimb by proprioceptive spinal pathways. In: Neurosurgery: state of the art reviews, pp 303-314. Philadelphia: Hanley and Belfus.

Poggio I, Girosi F (1990) A theory of networks for learning. Science 247:978-982.

Powell MJD (1987) Radial basis functions for multivariable interpolation: a review. In: Algorithms for approximation, pp. 143-167. Oxford: Clarendon.

Russell DL (1990) An analysis of constrained motions in manipulation. $\mathrm{PhD}$ thesis, Massachusetts Institute of Technology.

Shadmehr R (1991) A computational theory for control of posture and movement in a multi-joint limb. PhD thesis, University of Southern California, Los Angeles.

Smith AM, Humphrey DR (1991) What do studies of specific motor acts such as reaching and grasping tell us about the general principles of goal-directed motor behavior? In: Motor control: concepts and issues (Humphrey DR, Freund H-J, eds), pp 357-381. Chichester: Wiley.

Vince MA (1948) Corrective movements in a pursuit task. Q J Exp Psychol 1:85-106.

Vincken MH, Gielen CCAM, Denier van der Gon JJ (1983) Intrinsic and afferent components in apparent muscle stiffness in man. Neuroscience 9:529-534.

von Mises R (1964) Mathematical theory of probability and statistics. New York: Academic. 